\title{
A Robust Timing and Frequency Synchronization for OFDM Systems
}

\author{
Hlaing Minn, Member, IEEE, Vijay K. Bhargava, Fellow, IEEE, and Khaled Ben Letaief, Fellow, IEEE
}

\begin{abstract}
A robust symbol-timing and carrier-frequency synchronization scheme applicable to orthogonal frequency-division-multiplexing systems is presented. The proposed method is based on a training symbol specifically designed to have a steep rolloff timing metric. The proposed timing metric also provides a robust sync detection capability. Both time domain training and frequency domain (FD) training are investigated. For FD training, maintaining a low peak-to-average power ratio of the training symbol was taken into consideration. The channel estimation scheme based on the designed training symbol was also incorporated in the system in order to give both fine-timing and frequency-offset estimates. For fine frequency estimation, two approaches are presented. The first one is based on the suppression of the interference introduced in the frequency estimation process by the training symbol pattern in the context of multipath dispersive channels. The second one is based on the maximum likelihood principle and does not suffer from any interference. A new performance measure is introduced for timing estimation, which is based on the plot of signal to timing-error-induced average interference power ratio against the timing estimate shift. A simple approach for finding the optimal setting of the timing estimator is presented. Finally, the sync detection, timing estimation, frequency estimation, and bit-error-rate performance of the proposed method are presented in a multipath Rayleigh fading channel.
\end{abstract}

Index Terms-Frequency-offset estimation, orthogonal frequency-division multiplexing (OFDM), symbol-timing estimation, synchronization, training symbol.

\section{INTRODUCTION}

$\mathbf{O}$ RTHOGONAL frequency-division multiplexing (OFDM) has been of major interest for both wireline-based and wireless applications [1]-[3] due to its high data rate transmission capability and its robustness to multipath delay spread. However, OFDM systems are much more sensitive to synchronization errors than single carrier systems [4], [5]. Several approaches have been proposed for estimating the time and frequency offset either jointly [6]-[8] or individually. See [9]-[12] for further notes on frequency-offset estimation

Manuscript received June 19, 2001; revised January 17, 2002; accepted March 5, 2002. The editor coordinating the review of this paper and approving it for publication is L. Hanzo. This work was supported in part by a Strategic Project Grant from the Natural Sciences and Engineering Research Council (NSERC) of Canada and in part by TELUS Mobility Cellular.

H. Minn is with the Erik Jonsson School of Engineering and Computer Science, University of Texas at Dallas, Richardson, TX 75083-0688 USA (e-mail: hlaing.minn@utdallas.edu).

V. K. Bhargava is with the Department of Electrical and Computer Engineering, University of British Columbia, Vancouver, BC V6T 1Z4, Canada (e-mail: v.bhargava@ieee.org).

K. B. Letaief is with the Center for Wireless Information Technology, Department of Electrical and Electronic Engineering, The Hong Kong University of Science and Technology, Clear Water Bay, Kowloon, Hong Kong (e-mail: eekhaled@ee.ust.hk).

Digital Object Identifier 10.1109/TWC.2003.814346 and to [13]-[16] on timing estimation. There are of numerous other relevant contributions in the literature and a good discussion on them can be found in the recent paper [28].

Most frequency and timing estimation methods exploit the periodic nature of the time-domain signal by using a cyclic prefix [6], [8], [10], [13], or by designing the training symbol having repeated parts [7], [9], [12]. Regarding frequency estimation, in [7], a training symbol containing two identical halves is used and the frequency acquisition range is \pm 1 subcarrier spacing. In order to increase the frequency capture range, a second training symbol is employed. A frequency estimation scheme improving the solution of [7] is proposed in [12], where only one training symbol having $L$ identical parts is required and the frequency acquisition range is $\pm L / 2$ subcarrier spacing. A robust timing synchronization scheme using a training symbol having two identical parts has also been proposed in [7]. However, the timing metric plateau inherent in [7] results in a large timing offset estimation variance. This timing metric plateau can be eliminated and, hence, the timing offset estimation variance can be reduced by designing the training symbol such that it gives a more pronounced timing metric trajectory [15]. For single carrier systems, in [17], the frame synchronization performance has been considerably improved by designing the training preambles to give a sharper peak timing metric trajectory.

All of the above OFDM synchronization methods are associated with one or more of the following limitations or drawbacks: have a limited range of operation, address only one task, have a large estimation variance, lack robust sync detection capability, and require extra overheads. To overcome these limitations, we investigate both timing and frequency synchronization for OFDM, particularly using only one training symbol (or one training block) in this paper. In this case, the training symbol is designed to have a sharp timing metric trajectory. In choosing the timing metric, a robust sync detection capability has to be taken into consideration. We design the training symbol to be composed of repeated identical parts with possible sign inversions. Our choice of this type of training symbol is based on the following reasons. The periodicity or repetitive nature of the training symbol equips timing synchronization with robustness against frequency offsets. Having multiple identical parts gives the benefit of using the same training symbol for frequency synchronization, which can handle large frequency offsets. By designing the signs of the identical parts to give the sharpest possible timing metric trajectory, the timing offset estimation can be improved. Both OFDM-type training symbols (frequency domain (FD) training) and single-carrier-type training blocks (time domain (TD) training) are investigated. 
We present a synchronization scheme, which provides both timing and frequency estimation as well as channel estimates. A specifically designed training symbol is used for both timing and frequency synchronization. Channel estimation based on the designed training symbol is also incorporated in order to give fine-timing and frequency-offset estimates. Fine synchronization can also be iteratively improved. The impact of using only one training symbol for both timing and frequency synchronization is discussed and a number of approaches are proposed for further performance improvement. The sync detection performance, timing synchronization performance, frequency synchronization performance, and bit-error-rate (BER) performance of the proposed method is evaluated by computer simulations.

Regarding our timing estimation performance measure in the context of OFDM, the additional interference power caused by timing estimation might be considered, rather than the timing offset estimation variance, since the former reflects the actual impact of timing synchronization error on the system's performance. However, the interference power may also depend on the mean of the timing estimate. Hence, in this paper, we introduce a more revealing performance measure for the timing characterization of OFDM systems.

This paper is organized as follows. Section II describes the system considered. Section III briefly presents the OFDM synchronization problem and the effects of synchronization errors. In Section IV, the proposed synchronization scheme is presented. Performance evaluation, simulation results, and discussions are provided in Section V. Finally, our conclusions are provided in Section VI.

\section{SYSTEM DESCRIPTION}

The samples of the transmitted baseband OFDM signal, assuming ideal Nyquist pulse shaping, can be expressed as

$$
s(k)=\frac{1}{\sqrt{N}} \sum_{n=0}^{N_{u}-1} c_{n} \exp (j 2 \pi k n / N), \quad-N_{g} \leq k \leq N-1
$$

where $c_{n}$ is the modulated data or subcarrier symbol, $N$ is the number of inverse fast Fourier transform (IFFT) points, $N_{u}(\leq N)$ is the number of subcarriers, $N_{g}$ is the number of guard samples, and $j=\sqrt{-1}$. Consider a frequency selective multipath fading channel with path gains $\left\{h_{l}: l=0,1, \ldots, K-1\right\}$ (including possible paths with a zero gain) and the corresponding path delays $\left\{\tau_{l}\right\}$. We assume that the path delays are sample-spaced (namely, $\tau_{l}=l$ ). Hence, $\left\{h_{l}\right\}$ represents the discrete-time channel impulse response.

At the receiver, there exist carrier-frequency offset, sampling clock errors, and symbol-timing offset, which have to be estimated and compensated. Usually, the frequency offset and timing errors are more dominant than the sampling clock inaccuracy. Hence, we will consider, in this paper, the carrier-frequency and symbol-timing synchronization, assuming a perfect sampling clock. In this case, the received samples become

$$
r(k)=\exp (j \phi) \exp (j 2 \pi k v / N) \sum_{l=0}^{K-1} h_{l} s\left(k-\tau_{l}\right)+n(k)
$$

where $n(k)$ is the sample of zero-mean complex Gaussian noise process with variance $\sigma_{n}^{2}, v$ is the carrier-frequency offset normalized by the subcarrier spacing, and $\phi$ is an arbitrary carrier phase factor. The timing point of the start of the FFT window is determined by the timing synchronization scheme to be at the sample $r(\varepsilon)$ where $\varepsilon$ is a timing offset in units of OFDM samples. In this paper, we consider only integer timing offsets. If noninteger timing offsets and/or sampling clock errors need to be considered for a particular system, the method of [16] may be applied.

\section{SYNCHRONIZATION}

In OFDM systems, the main synchronization parameters to be estimated are a sync flag indicating the presence of the signal (especially for burst-mode transmission), the starting time of the FFT window (timing synchronization), the frequency offset due to the inaccuracies of the transmitter and receiver oscillators, and the Doppler shift of the mobile channel, as well as the channel estimates if coherent reception is adopted. The sync flag can be generated by automatic gain control (e.g., ramp-up indication via power measurement and threshold decision) or using a training symbol (which can also be used for timing synchronization and possibly frequency synchronization). For the latter case, the same metric used for timing synchronization may be used together with the threshold decision, in order to generate the sync flag. After detecting the presence of the signal, the other sync parameters are estimated. In the following, the effect of timing synchronization errors is briefly described for the later use.

\section{A. Effect of Timing Offset}

Let the sample indexes of a perfectly synchronized OFDM symbol be $\left\{-N_{g}, \ldots,-1,0,1, \ldots, N-1\right\}$, the timing offset be $\varepsilon$, and the maximum channel delay spread be $\tau_{\max }$. Then, if $\varepsilon \in\left\{-N_{g}+\tau_{\max },-N_{g}+\tau_{\max }+1, \ldots, 0\right\}$, the orthogonality among the subcarriers will not be destroyed and the timing offset will only introduce a phase rotation in every subcarrier symbol $Y_{m}$ at the FFT output as

$Y_{m}=\exp (j 2 \pi m \varepsilon / N) c_{m} H_{m}+n_{m}, \quad-N_{g}+\tau_{\max } \leq \varepsilon<0$

where $m$ is the subcarrier index, $H_{m}$ is the channel's frequency response for the $m$ th subchannel, i.e., $\left\{H_{m}\right\}=\operatorname{DFT}_{N}\left(h_{l}\right)$, and $n_{m}$ is a complex Gaussian noise term. For a coherent system, this phase rotation is compensated by the channel equalization scheme, which views it as a channel-induced phase shift. If the timing estimate is outside the above range, the orthogonality among the subcarriers will be destroyed by the resulting intersymbol interference (ISI) and additional intersubchannel interference (ICI) will be introduced as [21]

$$
Y_{m}=\exp (j 2 \pi m \varepsilon / N) \alpha(\varepsilon) c_{m} H_{m}+I_{m}+n_{m}
$$

where

$$
\alpha(\varepsilon) \simeq \sum_{l}\left|h_{l}(t)\right|^{2} \frac{N-\Delta \varepsilon_{l}}{N}
$$




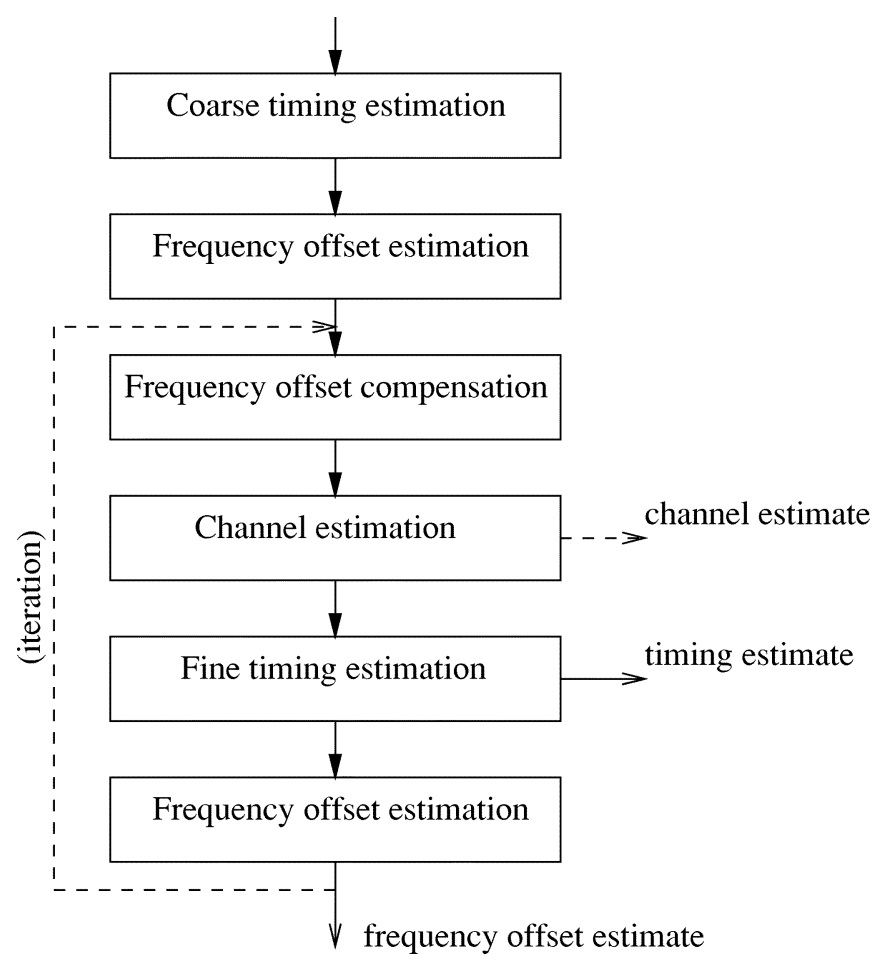

Fig. 1. Proposed synchronization scheme.

and $I_{m}$ constitutes both ISI and ICI terms. These terms can be modeled as Gaussian noise having a power of (for unit signal power on each subcarrier)

$$
\sigma_{\varepsilon}^{2}=\sum_{l}\left|h_{l}(t)\right|^{2}\left(2 \frac{\Delta \varepsilon_{l}}{N}-\left(\frac{\Delta \varepsilon_{l}}{N}\right)^{2}\right)
$$

where for $\varepsilon>0$

$$
\Delta \varepsilon_{l}= \begin{cases}\varepsilon-\tau_{l}, & \varepsilon>\tau_{l} \\ \tau_{l}-N_{g}-\varepsilon, & 0<\varepsilon<-\left(N_{g}-\tau_{l}\right) \\ 0, & \text { else. }\end{cases}
$$

Similarly, for $\varepsilon<-N_{g}+\tau_{\max }$, we can have

$$
\Delta \varepsilon_{l}= \begin{cases}-N_{g}+\tau_{l}-\varepsilon, & \varepsilon<-N_{g}+\tau_{l} \\ 0, & \text { else. }\end{cases}
$$

Hence, the guard interval should be long enough for the timing estimate to lie within the above range. In a loose sense, the smaller the variance of the timing estimator, the shorter the guard interval, and, hence, the lower the overhead. For the effect of carrier-frequency offset, see [21].

\section{PRoposed SynCHRONIZATION METHOD}

The scheme proposed for symbol-timing and frequency synchronization of OFDM systems is shown in Fig. 1 where a specifically designed training symbol is used. The sync flag is determined by the timing metric and an associated threshold decision. In the following, we assume that the presence of the signal has already been detected and, hence, the rest of the synchronization part will be presented. First, coarse-timing estimation is performed based on the timing metric. It gives the estimate of the start position of the FFT window for the training symbol. The frequency offset is estimated based on the training symbol defined by the coarse-timing estimation. Frequency-offset compensation is then performed on the training symbol. Next, the channel impulse response is estimated based on the frequency offset compensated received training symbol. Given the channel estimation, the delay of the first channel path is found and added to the coarse-timing estimate to give a fine-timing estimate. The new training symbol defined by the fine-timing estimate is used to estimate the fine frequency offset. Hence, the fine synchronization part contains frequency-offset compensation, channel impulse response estimation, fine-timing offset estimation, and fine frequency-offset estimation. This fine synchronization procedure can be repeated in order to achieve further improvements. The channel impulse response can then be estimated again after performing frequency-offset compensation on the training symbol defined by the fine-timing estimate. It may be directly used or further processed for employment in channel equalization, but further processing on the channel response estimate will not be considered in this paper. It is also noted that the proposed synchronization method is applicable to both continuous-mode transmission and burst-mode transmission since it does not utilize any property specific to a particular transmission mode such as null interval in the burst-mode transmission. In our simulations, we use a burst-mode transmission where the training symbol is preceded by noise samples and followed by data symbols.

\section{A. Proposed Timing Metric}

A desirable property of a timing estimation scheme is its robustness to the frequency offsets. If the training symbol has a repetitive structure, a timing estimation scheme which is robust to frequency offset can be obtained by means of correlation among the repetitive parts. Hence, we consider a repetitive training structure in this paper. Assuming that the training symbol is composed of two identical parts of $M$ samples each, then this type of training symbol with two repeated parts can be efficiently searched by minimizing the metric which calculates the squared averaged distance between the two considered received signal parts of length $M$ samples each as [18], [19]

$$
\mathcal{V}(d)=\frac{E(d)}{M}-\frac{2}{M} \cdot|P(d)|
$$

where

$$
\begin{aligned}
& E(d)=\sum_{i=0}^{M-1}\left(|r(i+d+M)|^{2}+|r(i+d)|^{2}\right) \\
& P(d)=\sum_{i=0}^{M-1} r(i+d+M) \cdot r^{*}(i+d) .
\end{aligned}
$$

It can be easily observed that the above metric is robust to any frequency offset. This minimum mean square error (MMSE)-type metric (9) shows almost the same timing estimation performance as the maximum likelihood (ML) estimate. (For detailed timing performance comparison, see [20].) However, the above metric and those in [20], except the Schmidl and Cox (S\&C) metric of [7], do not consider sync detection and are associated with a high false detection probability. This fact can be observed from (9) as follows. Under a 
noiseless condition, $\mathcal{V}(d)$ will give a minimum metric value of zero when $d$ is at the correct timing point and approximately a maximum metric value of $E(d) / M$ when $d$ is such that $\{r(d+i): i=0,1, \ldots, N-1\}$ do not correspond to the training symbol. When only noise is present, $\mathcal{V}(d)$ would give some value close to zero since the corresponding $E(d) / M$ would be very small, hence, causing a false detection. Similar result will be obtained when the transmitted data symbols are in deep fade. This high false detection probability can be reduced using the normalized metric

$$
\mathcal{V}_{n}(d)=\frac{E(d)-2|P(d)|}{E(d)} .
$$

Finding the minimum of $\mathcal{V}_{n}(d)$ is equivalent to finding the maximum of

$$
\Lambda_{n}(d)=\frac{2}{E(d)} \cdot|P(d)| .
$$

Under a noiseless condition, $\Lambda_{n}(d)$ will give a maximum value of one when $d$ is at the correct timing point and approximately a minimum value of zero when $d$ is such that $\{r(d+i): i=$ $0,1, \ldots, N-1\}$ do not correspond to the training symbol. When only noise is present, $|P(d)|$ would be approximately equal to zero and would be much less than $E(d)$. Hence, $\Lambda_{n}(d)$ would give a value close to zero and a false detection would be avoided. Similar results will hold when the transmitted data symbols are in deep fade.

In practice, $\Lambda_{n}^{2}(d)$ instead of $\Lambda_{n}(d)$ can be considered. The timing metric of (13) which was introduced in the context of two identical parts can be further generalized for a training symbol containing $L$ rather than two parts of $M$ samples each. In this case, the timing metric to be maximized can be expressed as

$$
\Lambda_{\varepsilon}(d)=\left(\frac{L}{L-1} \frac{|P(d)|}{E(d)}\right)^{2}
$$

where

$$
\begin{aligned}
P(d)= & \sum_{k=0}^{L-2} b(k) \cdot \sum_{m=0}^{M-1} r^{*}(d+k M+m) \\
& \cdot r(d+(k+1) M+m) \\
E(d)= & \sum_{i=0}^{M-1} \sum_{k=0}^{L-1}|r(d+i+k M)|^{2}
\end{aligned}
$$

and $b(k)=p(k) p(k+1), k=0,1, \ldots, L-2$.

In the above equation, $\{p(k): k=0,1, \ldots, L-1\}$ denote the signs of the repeated parts of the training symbol and will be called the training symbol pattern in the rest of this contribution. For $L=2$ and for the training symbol pattern $p=[++]$, the timing metric of $\mathrm{S} \& \mathrm{C}$ is obtained if $E(d)$ is approximated by $2 R(d)$ where $R(d)=\sum_{i=0}^{M-1}|r(i+d+M)|^{2}$. Hence, the $\mathrm{S} \& \mathrm{C}$ timing metric can be considered as an approximation of the proposed metric for the case of $L=2$ and $p=[++]$.

\section{B. Training Symbol}

The training symbol can be designed either for an OFDM-type FD training or single-carrier-type TD training [27]. The training symbol of $\mathrm{S} \& \mathrm{C}$ is of the FD-type and gives a timing metric plateau which results in a high timing estimator variance. This can be avoided by designing the training symbol to have a steep rolloff timing metric trajectory. For example, the HiperLAN/2-type training symbol was designed for providing a steep rolloff timing metric trajectory [22]. On the other hand, it does not preserve the cyclic prefix structure of an OFDM system. In this paper, we will consider a training symbol that preserves the cyclic prefix structure. We design the training symbol such that it is composed of $L$ identical parts to handle a frequency offset of up to $\pm L / 2$ subcarrier spacing and has a specific pattern (signs) of the $L$ identical parts to give a timing metric having a steep rolloff trajectory. In our context, S\&C uses an FD-type training with $L=2$ and the pattern of $[++]$, while Morelli and Mengali (M\&M) [12] use an FD-type training with $(L>2)$ and the pattern of all + signs.

In order to avoid nonlinear distortion at the transmitter, the training symbol should be designed to have a low peak-to-average power ratio (PAPR). Golay complementary sequences [23] are well known for having specific correlation properties which translate into a low PAPR value $(3 \mathrm{~dB})$ when they are mapped to the OFDM subcarriers [24]. Hence, a Golay complementary sequence is applied in our FD training symbol as follows. The repeated part has $M=N / L$ samples (for $N$ point IFFT and $L$ identical parts), and it is generated by the $M$-point IFFT of a length $N_{u} / L$ Golay complementary sequence. By contrast, for TD training, a length $M$ Golay complementary sequence is directly used in the TD as the basic repeated part. In this case, it represents a constant amplitude training sequence. However, the cyclic prefix extension based structure is still maintained in the context of TD training. As an example, let $N=N_{u}=64, L=4$, the bipolar representation of Golay complementary sequence of length 16 be $C$, and the time-domain repeated part of length 16 be $\boldsymbol{A}$. Then, for TD training $\boldsymbol{A}=\boldsymbol{C}$ and for FD training $\boldsymbol{A}=F F T_{16}(\boldsymbol{C})$. In Fig. 2, the corresponding time-domain samples of the training symbol (excluding cyclic prefix) with the pattern $[-+--]$, which can be given by $\left[\begin{array}{llll}-\boldsymbol{A} & \boldsymbol{A}-\boldsymbol{A}-\boldsymbol{A}\end{array}\right]$, are presented. The repeated part boundaries are indicated by dashed lines.

1) Training Symbol Pattern: Suppose that the training symbol (excluding cyclic prefix) is composed of $L$ identical parts as $[ \pm \mathbf{A}, \pm \mathbf{A}, \ldots, \pm \mathbf{A}]$, where $\mathbf{A}$ represents the repeated part. If the timing metric given in (14) and (15) is used, then the best patterns (signs of the repeated parts) of the training symbol obtained by computer search are given in Table I for $10 \%$ cyclic prefix, and $L=4,8$, and 16 . These patterns were obtained by finding the patterns which give a minimum value of $\sum_{d=-N}^{N}|P(d)|^{2}$. In the computer search, $\left\{r(k):-N_{g} \geq k \leq N-1\right\}$ correspond to the training symbol and the other $\{r(k)\}$ are simply set to zero. In Fig. 3(a), the plots of $|P(d)|^{2}$ for all possible patterns with $L=4$ are shown. The patterns can be expressed as the bipolar form of the binary representation of the pattern sequence numbers shown on the subplots. From Fig. 3(a), it can be observed that the best pattern sequence numbers are 2, 7, 8, and 13. In Fig. 3(b), the corresponding values of $\sum_{d=-N}^{N}|P(d)|^{2}$ are shown for all pattern sequence numbers. The pattern sequence numbers $2,7,8$, and 13 have the minimum value. It can be observed that the minimum values generally correspond to the best 
(a)

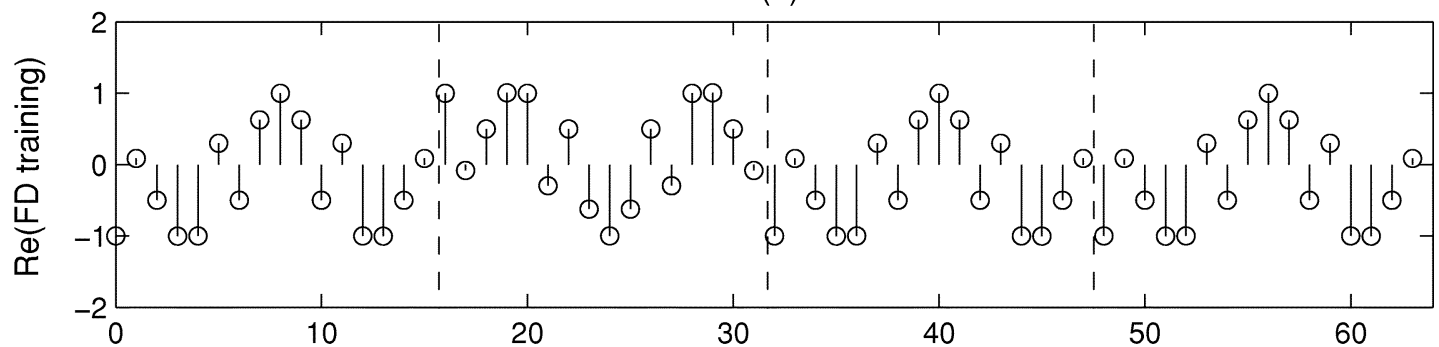

(b)

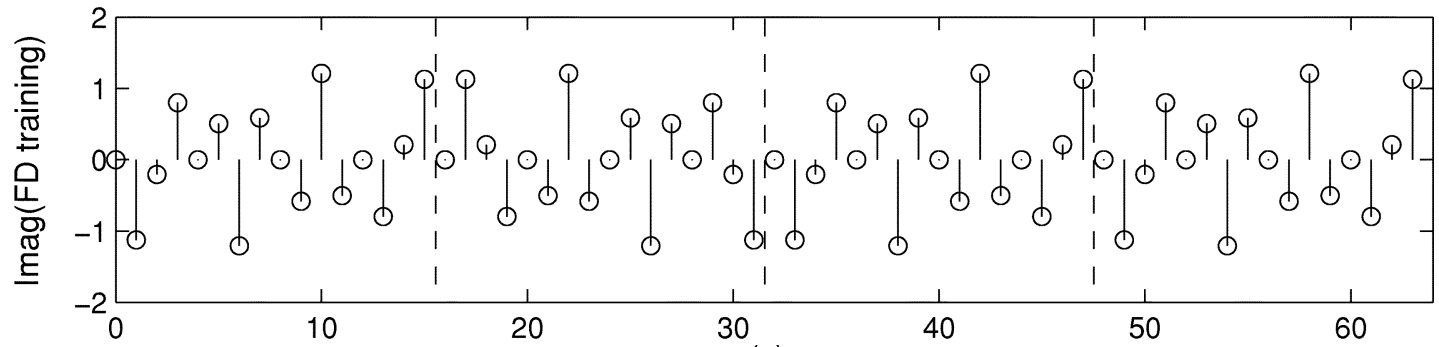

(c)

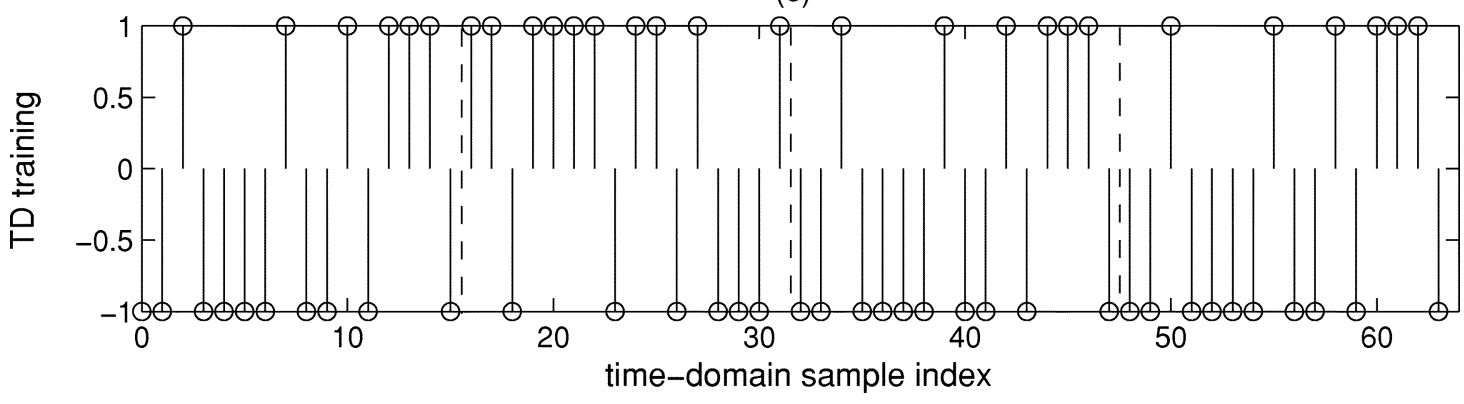

Fig. 2. Example of the time-domain samples of the 64-sample length training symbol defined by $[-\boldsymbol{A} A-\boldsymbol{A}-\boldsymbol{A}]$. (The corresponding training symbol pattern is $[-+--]$. The cyclic prefix part is not shown.) (a) Real part for FD training. (b) Imaginary part for FD training. (c) TD training.

TABLE I

TRAINING SYMBOL PATTERN

\begin{tabular}{c|c}
\hline$L$ & Pattern \\
\hline 4 & $(-+--)$ \\
& $(+++-)$ \\
\hline 8 & $(++--+---)$ \\
& $(-++---+-)$ \\
\hline 16 & $(+--+++--+-++-+--)$ \\
& $(--++-++---++++-)$ \\
\hline
\end{tabular}

steepest rolloff correlation metric trajectories. Each $L$ value has four patterns. The first two are shown in Table I, and the other two not shown are the sign-inversion based variants of the first two. The trajectories of the timing metrics used in coarse-timing estimation for the training symbol patterns (the first one for each $L$ value) given in Table I are shown in Fig. 4 under noiseless and no channel distortion condition. The timing metric trajectory of $\mathrm{S} \& \mathrm{C}$ is also included for comparison. Unlike $\mathrm{S} \& \mathrm{C}$, there are no timing metric trajectory plateaus associated with the proposed method. A larger value of $L$ gives a timing metric trajectory with a steeper rolloff.

\section{Coarse-Timing Estimation}

Coarse-timing estimation is based on the correlation among the $L$ parts of size $M$ samples each. The coarse-timing estimator takes as the start of OFDM symbol (after cyclic prefix) the maximum point $d_{\max }$ of the timing metric given by (14)-(16), where $d$ is a time index corresponding to the first sample in a window of $N$ samples. In order to maintain orthogonality among subcarriers, the timing estimate should be in the ISI-free part of the cyclic prefix (i.e., the sample indexes $-N_{g}+\tau_{\max },-N_{g}+$ $\left.\tau_{\max }+1, \ldots, 0\right)$. In an additive white Gaussian noise (AWGN) channel, the mean of the proposed timing metric trajectory peak is at the exact timing point, but in multipath channels it would be shifted (delayed) due to the channel dispersion. Hence, the coarse-timing estimate $\hat{\varepsilon}_{c}$ should be preadvanced by some samples $\lambda_{c}$ as

$$
\hat{\varepsilon}_{c}=d_{\max }-\lambda_{c}
$$

where $\lambda_{c}$ should be chosen to be higher than the (designed) mean shift of the timing point caused by the channel dispersion.

\section{Coarse Carrier-Frequency Offset Estimation}

For coarse carrier-frequency estimation, we follow the method of M\&M [12] with appropriate modifications. Since the only difference in the training symbol structure is the sign 
(a)
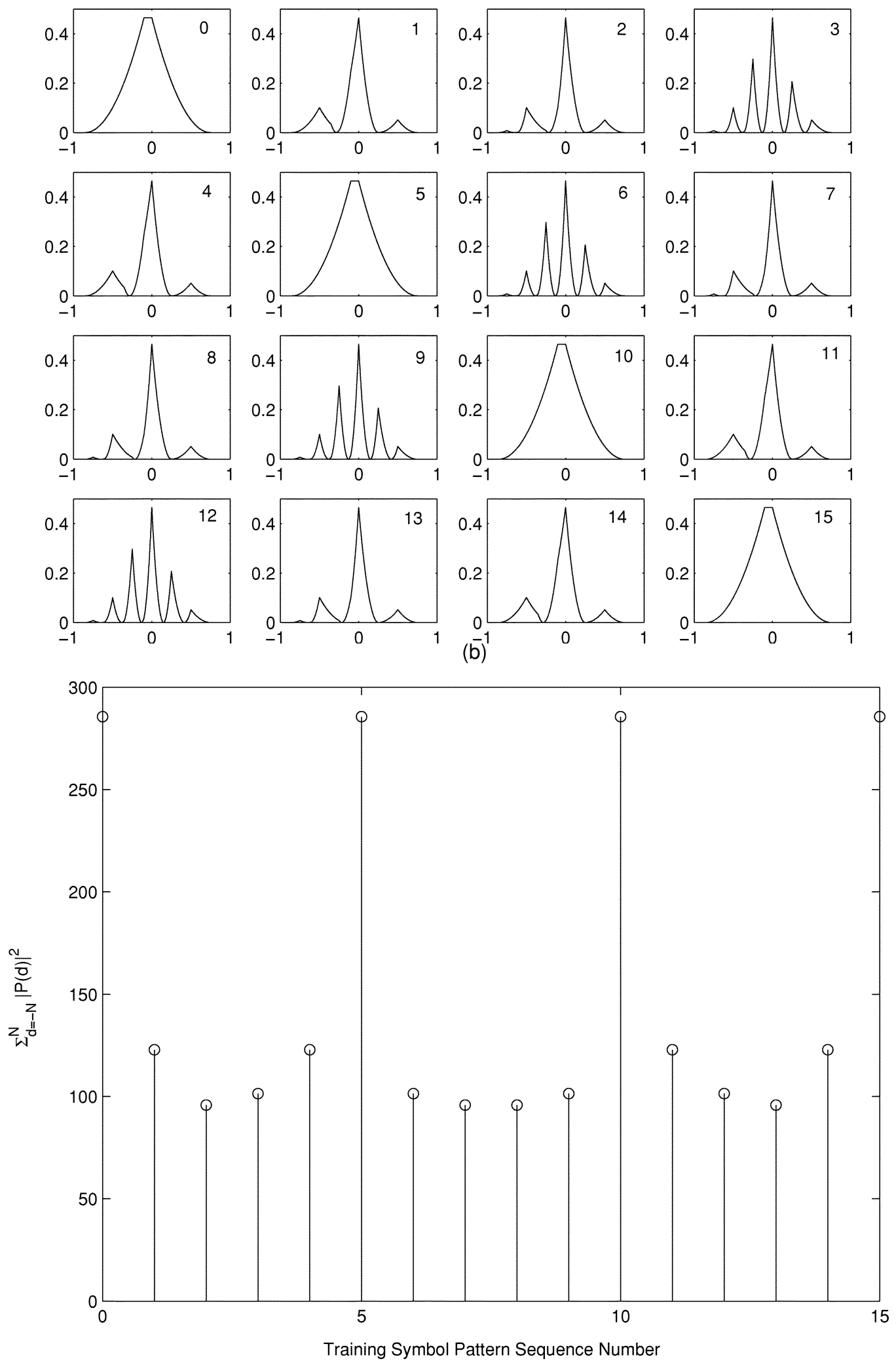

Fig. 3. (a) Plot of $|P(d)|^{2}$ versus $d / N$ corresponding to all possible patterns for $L=4$. The patterns can be expressed as the bipolar representation of the sequence number shown on the subplots. (b) The corresponding plot of $\sum_{d=-N}^{N}|P(d)|^{2}$ versus all patterns denoted by the sequence numbers.

pattern of $L$ identical parts, the training symbol defined by the timing estimator is first modified to have the same structure as that proposed in M\&M by multiplying the $L$ parts with the sign pattern applied in the training symbol design. Then, the method of M\&M is applied as follows. Let the modified training symbol defined by the coarse-timing estimate be 


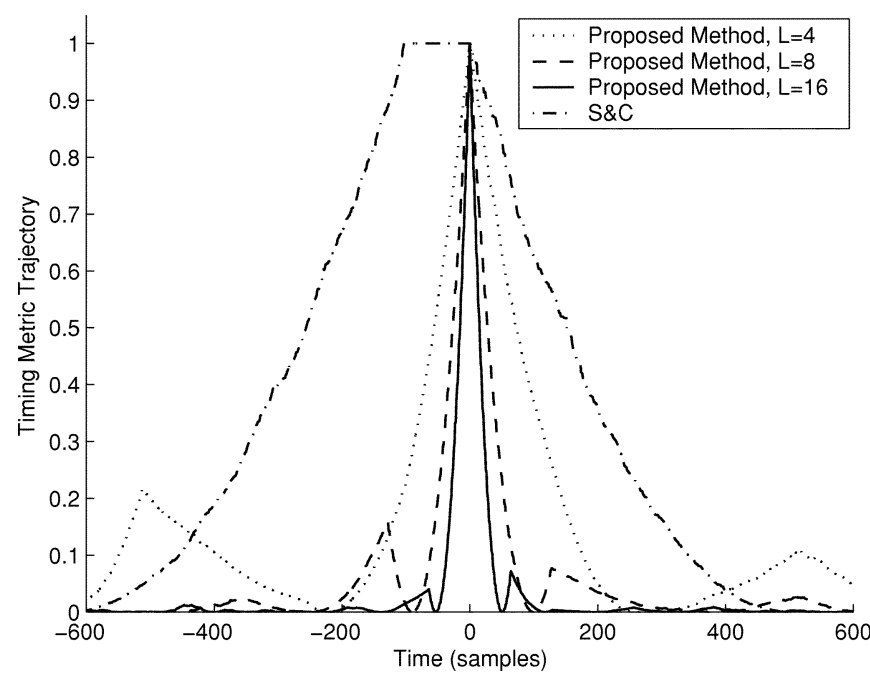

Fig. 4. Timing metric trajectory under noiseless conditions assuming no channel distortion. (Time index 0 corresponds to the exact timing point.)

represented by $\{y(k): k=0,1, \ldots, N-1\}$. Then, the coarse frequency-offset estimator is given by

$$
\hat{v}=\frac{L}{2 \pi} \sum_{m=1}^{H} w(m) \varphi(m)
$$

where

$$
\begin{gathered}
w(m)=3 \frac{(L-m)(L-m+1)-H(L-H)}{H\left(4 H^{2}-6 L H+3 L^{2}-1\right)} \\
\varphi(m)=\left[\arg \left\{R_{y}(m)\right\}-\arg \left\{R_{y}(m-1)\right\}\right]_{2 \pi} \\
1 \leq m \leq H
\end{gathered}
$$

$R_{y}(m)=\frac{1}{N-m M} \sum_{k=m M}^{N-1} y^{*}(k-m M) y(k), \quad 0 \leq m \leq H$

and $[x]_{2 \pi}$ denotes modulo $2 \pi$ operation (it reduces $x$ to the interval $[-\pi, \pi)$ ), $\arg \left\{R_{y}(m)\right\}$ is argument of $R_{y}(m)$, and $H$ is a design parameter less than or equal to $L-1$. The optimal value for $H$ is $L / 2$ which will be used in our approach.

The frequency-offset estimation range is $\pm L / 2$ subcarrier spacing for a training symbol having $L$ identical parts. However, the range is not limited by the length $L$ of the sign pattern in our design. For example, by designing the repeated part having $k$ identical subparts and using $H=L k / 2$ and $M=N /(L k)$, the range becomes $\pm L k / 2$. This is because the range depends on the spacing $M$ between the correlating identical parts and is defined by $\pm N /(2 M)$.

In a multipath dispersive channel, the repeated parts of the received training symbol will not be equal, even in the absence of noise, due to the sign conversion in the transmitted training symbol. This effect perturbs the repetitive nature of the received training symbol. For frequency estimation, the received training symbol is multiplied with the sign pattern to restore the repetitive parts of all equal sign, but this sign flipping will not remedy the already channel-impaired repetitive parts. Hence, some interference is introduced to frequency estimation. Suppression of this interference will be addressed in the fine frequency-offset estimation.

\section{E. Channel Impulse Response Estimation}

Assume that the channel response remains constant over at least one OFDM symbol interval (quasi-static case), and let the instantaneous path gains be $h_{0}, h_{1}, \ldots, h_{K-1}$. Let us define the following:

$$
\begin{aligned}
\mathbf{r}(0) & \triangleq\left[\begin{array}{llll}
r(0) & r(1) & \cdots & r(N-1)
\end{array}\right]^{T} \\
\mathbf{h} & \triangleq\left[\begin{array}{llll}
h_{0} & h_{1} & \cdots & h_{K-1}
\end{array}\right]^{T} \\
\mathbf{W}(v) & \triangleq \operatorname{diag}\left\{1, e^{j 2 \pi v / N}, e^{j 2 \pi 2 v / N}, \ldots, e^{j 2 \pi(N-1) v / N}\right\} \\
\mathbf{n} & \triangleq\left[\begin{array}{lllll}
n(0) & n(1) & \cdots & n(N-1)
\end{array}\right]^{T} \\
\mathbf{S} & \triangleq\left[\begin{array}{cccc}
s(0) & s(-1) & \cdots & s(-K+1) \\
s(1) & s(0) & \cdots & s(-K+2) \\
\ddots & & & \\
s(N-1) & s(N-2) & \cdots & s(N-K)
\end{array}\right]
\end{aligned}
$$

where $\left\{s(k): k=-N_{g},-N_{g}+1, \ldots, N-1\right\}$ are the samples of the transmitted training symbol (including the cyclic prefix part), $\{r(k): k=0,1, \ldots, N-1\}$ the corresponding received samples (excluding the cyclic prefix part), $\{n(k): k=$ $0,1, \ldots, N-1\}$ the noise samples, and $v$ the normalized frequency offset. The received sample vector can then be expressed as

$$
\mathbf{r}(0)=e^{j \phi} \mathbf{W}(v) \cdot \mathbf{S} \cdot \mathbf{h}+\mathbf{n} .
$$

Utilizing the frequency-offset estimate $\hat{v}$ in place of $v$, the ML channel response estimate [25] can be realized by

$$
\hat{\mathbf{h}}=\left[\mathbf{S}^{H} \cdot \mathbf{S}\right]^{-1} \mathbf{S}^{H} \cdot \mathbf{W}^{H}(\hat{v}) \cdot \mathbf{r}(0)
$$

where $(\cdot)^{H}$ represents a Hermitian transpose, $(\cdot)^{-1}$ represents a generalized matrix inverse, and the phase factor has been absorbed in the channel estimate.

In the above channel estimation, the knowledge of the maximum channel delay spread is required. In practice, a design value $K^{\prime}$ has to be used. Moreover, due to the timing estimation error and the timing advance introduced in Section IV-C, the received training vector will be $\mathbf{r}(\varepsilon)$. The advancement of the timing offset estimate should be adjusted such that $\varepsilon$ becomes negative most of the time. Otherwise, it will not only introduce ISI, but also miss some channel taps in the channel estimation and cause large channel estimation errors. Consequently, the designed channel estimate length should be longer than the designed maximum channel delay spread plus the delay introduced by timing estimate advancement. Let the designed channel estimate length be $K^{\dagger}$ (which may be set to the guard interval length). By replacing $K$ with $K^{\dagger}$ in (22), the channel response estimate is given by

$$
\hat{\mathbf{h}}=\left[\mathbf{S}^{H} \cdot \mathbf{S}\right]^{-1} \mathbf{S}^{H} \cdot \mathbf{W}^{H}(\hat{v}) \cdot \mathbf{r}(\varepsilon)
$$

where $\mathbf{W}^{H}(\hat{v}) \cdot \mathbf{r}(\varepsilon)$ is the frequency-offset compensated received training vector defined by the timing estimate $\varepsilon$.

If the length of the basic repeated training symbol part $M$ is larger than the maximum channel delay spread, then the channel estimation complexity can be reduced by combining the basic repeated parts as follows. Let us consider $L=4$ and 
let the repeated parts of the received training symbol, after frequency-offset compensation, be $\left[\mathbf{Z}_{1} \mathbf{Z}_{\mathbf{2}} \mathbf{Z}_{3} \mathbf{Z}_{4}\right]$. Then, from the training symbol pattern $[-+--]$, it can be observed that $\mathbf{Z}_{\mathbf{1}}$ and $\mathbf{Z}_{\mathbf{4}}$ are equivalently affected by the multipath fading environment. Similarly, $-\mathbf{Z}_{\mathbf{2}}$ and $\mathbf{Z}_{3}$ are equivalently affected. Averaging over each set yields $\mathbf{Z}_{A}=\left(\mathbf{Z}_{\mathbf{1}}+\mathbf{Z}_{\mathbf{4}}\right) / 2$ and $\mathbf{Z}_{B}=\left(-\mathbf{Z}_{\mathbf{2}}+\mathbf{Z}_{\mathbf{3}}\right) / 2$. Note that the separate use of $\mathbf{Z}_{A}$ and $\mathbf{Z}_{B}$ is an impact of the training symbol pattern. Define $\mathbf{S}_{A}$ composed of $\left\{s\left(-K^{\dagger}+1\right), \ldots, s(M-1)\right\}$ as

$$
\mathbf{S}_{A} \triangleq\left[\begin{array}{cccc}
s(0) & s(-1) & \cdots & s\left(-K^{\dagger}+1\right) \\
s(1) & s(0) & \cdots & s\left(-K^{\dagger}+2\right) \\
\ddots & & & \\
s(M-1) & s(M-2) & \cdots & s\left(M-K^{\dagger}\right)
\end{array}\right] .
$$

Then, the channel estimate based on $\mathbf{Z}_{A}$ can be obtained as

$$
\hat{\mathbf{h}}_{A}=\left[\mathbf{S}_{A}^{H} \cdot \mathbf{S}_{A}\right]^{-1} \mathbf{S}_{A}^{H} \cdot \mathbf{Z}_{A}
$$

while $\hat{\mathbf{h}}_{B}$ based on $\mathbf{Z}_{B}$ can be obtained in a similar fashion using $\mathbf{S}_{B}$ composed of $\left\{s\left(2 M-K^{\dagger}+1\right), \ldots, s(3 M-1)\right\}$. The final channel estimate is taken as the average of $\hat{\mathbf{h}}_{A}$ and $\mathbf{h}_{B}$ as

$$
\hat{\mathbf{h}}=\left(\hat{\mathbf{h}}_{A}+\hat{\mathbf{h}}_{B}\right) / 2=\left\{\hat{h}_{i}, i=0,1, \ldots, K^{\dagger}-1\right\} .
$$

A similar approach can be applied to $L=8$ and 16 cases, if the length of the repeated part is much larger than the maximum channel delay spread. Throughout our simulations, (25) will be used for channel estimation.

\section{F. Fine-Timing Estimation}

The coarse-timing estimate would be, most of the time, before the actual timing point due to the preadvancement. However, even under noiseless conditions, the timing estimate would be varying according to the time-varying nature of the channel response. Accordingly, at different snap shots, the channel estimates would be delayed by different amounts due to different timing offset errors. If the delay in the channel estimate can be found, the effect of the time-varying channel response on the timing estimation can be removed by simply delaying the coarse-timing estimate by the same amount of the channel estimate delay. In other words, the coarse-timing estimate can be fine-tuned by adding the delay of the first actual channel tap from the channel estimate. A similar concept has been applied in [16], where some pilot tones are used throughout the burst (hence, FD-based channel estimation), $N_{\mathrm{av}}$ consecutive channel impulse response estimates are averaged, and the first channel tap is found by some criteria. In our considered system with one training symbol, the channel estimation is based on one snap-shot estimation in the TD, and a different criteria described in the following is used for the first channel tap selection.

One way of finding the delay of the first actual channel tap is described in the following. First, the strongest tap gain estimate $\hat{h}_{\text {max }}$ is found as $\hat{h}_{\max }=\max \left\{\hat{h}_{i}: i=0,1, \ldots, K^{\dagger}-1\right\}$. Then, the delay estimate of the first actual channel tap $\hat{\tau}_{0}$ is given by

$$
\hat{\tau}_{0}=\arg \max _{l}\left\{E_{h}(l): l=0,1, \ldots, K^{\dagger}-K^{\prime}\right\}
$$

where

$$
E_{h}(l)= \begin{cases}\sum_{k=0}^{K^{\prime}-1}\left|\hat{h}_{l+k}\right|^{2}, & \text { if }\left|\hat{h}_{l}\right|>\eta \cdot\left|\hat{h}_{\max }\right| \\ 0, & \text { otherwise. }\end{cases}
$$

$E_{h}(l)$ is the channel energy estimate contained in a length- $K^{\prime}$ window starting from the tap $l$ with a condition that the channel energy estimate of tap $l$ should be greater than some threshold. This condition reduces the probability of choosing a noise-only tap as the first actual channel tap. The delay estimate of the first actual channel tap given in (29) is essentially the starting tap index whose associated energy $E_{h}(l)$ is maximum. Due to the timing advancement $\lambda_{c}$ in the coarse stage, the actual channel impulse response would be delayed by some amount. Since the channel estimate has $K^{\dagger}$ taps and the designed maximum channel delay spread is $K^{\prime}$, the range of the starting tap index of the energy window is $0 \leq l \leq K^{\dagger}-K^{\prime}$, as described in (29). It should be noted that in order to keep the actual channel impulse response within the channel estimation length $K^{\dagger}, \lambda_{c}+K^{\prime}$ should be smaller than $K^{\dagger}$.

In the above equation, $\eta$ is a threshold factor for selecting the first channel tap. Note that $\eta$ should be small if we do not want to miss the first tap. However, $\eta$ should not be too small. Otherwise, the noise will increase the probability of wrongly picking up the channel tap before the first tap. One way for finding $\eta$ for different signal-to-noise ratio (SNR) values is given by (see the Appendix)

$$
\eta_{2}=\sqrt{\frac{\mathrm{SNR}_{1}}{\mathrm{SNR}_{2}}} \cdot \eta_{1}
$$

where $\eta_{1}$ is a known value obtained from the simulation at $\mathrm{SNR}_{1}$ and $\eta_{2}$ is the threshold value for $\mathrm{SNR}_{2}$.

The fine-timing estimate is obtained by adding the delay estimate of the first actual channel tap to the coarse-timing estimate as

$$
\hat{\varepsilon}=\hat{\varepsilon}_{c}+\hat{\tau}_{0}-\lambda_{f}
$$

where $\lambda_{f}$ is a designed preadvancement to reduce the possible ISI. Without the preadvancement of $\lambda_{f}$, the fine-timing estimate would be, most of the time, at the exact timing point. However, for the circumstances when the absolute gain ratio of the first tap to the strongest tap is smaller than $\eta$, the most likely chosen channel tap as the first tap would be the second channel tap. Hence, $\lambda_{f}$ should be at least the delay difference between the first and the second taps.

The preadvancement of fine-timing estimate may introduce interference in the frequency-offset estimation. The reason is that when the timing-advanced training symbol is multiplied by the training symbol pattern, the offset caused by the timing advancement would perturb the repeated structure of the training symbol and introduce some interference. Hence, a better choice of the received training symbol for frequency-offset estimation should be defined by the fine-timing estimate without preadvancement; namely, $\hat{\varepsilon}_{v}=\hat{\varepsilon}_{c}+\hat{\tau}_{0}$, and the fine-timing estimate is then advanced by $\lambda_{f}$, as in (32). In this way, the timing offset 
error interference in the frequency-offset estimation can be reduced and at the same time the ISI caused by the timing offset error can be reduced by means of preadvancement.

\section{G. Fine Frequency Estimation}

As mentioned in the coarse frequency estimation section, the training symbol pattern can introduce some interference to frequency estimation. If this interference is not taken into consideration in the fine stage, the fine frequency estimation will inherit the performance degradation. To suppress this interference, the differently affected received samples can be excluded from the frequency estimation. For $L=4$ and the training symbol pattern of $[-+--]$, it can be observed that after multiplying the received training symbol with the training symbol pattern, the first $(K-1)$ received samples of the second and third repeated parts are different from those of the other repeated parts, even in the absence of noise. Hence, they impair the repeated structure and introduce the interference. By excluding them, the interference can be suppressed. In practice, the first $K^{\prime}$ samples (designed maximum channel delay spread) of the second and third repeated parts may be excluded. The exclusion of those samples can easily be done by masking them with zeros. Then, the modified procedure becomes zero masking, sign flipping, and applying M\&M. This zero-masking approach can be applied if the number of samples in the basis part is much larger than the maximum channel delay spread.

Another approach to avoid the interference is based on the ML principle. Since fine-timing synchronization will most of the time give a perfect timing, we will assume that perfect timing has been achieved. By replacing $\boldsymbol{h}$ by the ML channel estimate (24), the ML frequency estimation can be obtained by maximizing the following metric:

$$
\Lambda\left(\hat{\varepsilon}_{v} ; \tilde{v}\right)=\boldsymbol{r}^{H}\left(\hat{\varepsilon}_{v}\right) \boldsymbol{W}(\tilde{v}) \boldsymbol{B} \boldsymbol{W}^{H}(\tilde{v}) \boldsymbol{r}\left(\hat{\varepsilon}_{v}\right)
$$

where $B=\boldsymbol{S}\left(\boldsymbol{S}^{H} \boldsymbol{S}\right)^{-1} \boldsymbol{S}^{H}$, and $\boldsymbol{S}$ is an $N \times K^{\prime}$ matrix. If $\hat{\varepsilon}_{v}=0$ and $K^{\prime}=K$, the above metric is exactly the same as that described in [26].

In the implementation of maximizing the metric, [26] used $N \cdot U$ point FFT in finding $\hat{v}$. Hence, the search space for $\hat{v}$ is approximately over the range of $(-N / 2, N / 2]$, and the resolution between trial frequency-offset points is $1 / U$ subcarrier spacing. In this paper, we make use of the information from the coarse frequency estimation. Since the coarse frequency-offset estimate cannot be too far away from the actual frequency offset, we limit the search space for $\hat{v}$ around the coarse frequency-offset estimate $\hat{v}_{c}$ as follows:

$$
\hat{v}=\arg \max _{\tilde{v}}\left\{\Lambda\left(\hat{\varepsilon}_{v} ; \tilde{v}\right): \hat{v}_{c}-F \leq \tilde{v} \leq \hat{v}_{c}+F\right\} .
$$

The trial points for $\tilde{v}$ are taken at a resolution of $\Delta=F / J$ subcarrier spacing within the search space; namely, $\{\tilde{v}(i)=$ $\left.\hat{v}_{c}+i \Delta: i=-J,-J+1, \ldots, J\right\}$, where $J$ is the number of trial points at one side of $\hat{v}_{c}$. The value of $F$ can be chosen according to the coarse frequency estimation performance. For example, if the error of the normalized coarse frequency-offset estimate is expected to be less than 0.01 (which is usually the case for good coarse frequency estimation methods at moderate or high SNR values), $F$ may be set to 0.01 or a little larger (say) 0.05 . Finally, the final frequency-offset estimate is given by

$$
\hat{v}_{f}= \begin{cases}\hat{v}, & \text { if } \hat{v}=\tilde{v}_{-J} \text { or } \tilde{v}_{J} \\ v_{q}, & \text { otherwise }\end{cases}
$$

where $v_{q}$ is obtained by quadratic interpolation among the points $\hat{v}-\Delta$, $\hat{v}$, and $\hat{v}+\Delta$.

\section{Performance Evaluation, Simulation RESULTS AND DISCUSSION}

\section{A. Simulation Parameters}

The performance of the proposed synchronization algorithm has been investigated by computer simulation. The OFDM system parameters used are 1024 subcarriers, 1024 point $I F F T / F F T$, and $10 \%$ guard interval (102 samples). The subcarrier modulation is quaternary phase-shift keying and a carrier-frequency offset of 6.2-subcarrier spacing is assumed. Unless stated otherwise, 10000 simulation runs will be applied. For the training symbol pattern, the first one of the two patterns given in Table I is used for each $L$ value.

The channels considered are described in the following. All channels except AWGN channel have 16 taps with equal tap spacing of four samples. The Rayleigh fading channel has an exponential power delay profile and the ratio of the first Rayleigh fading tap to the last Rayleigh fading tap is set to $20 \mathrm{~dB}$. The Rician fading channel has a Rician factor of four and the first tap with delay zero is set as the direct path. The other taps are Rayleigh fading taps with an exponential power delay profile and the ratio of the first Rayleigh fading tap to the last Rayleigh fading tap is set to $20 \mathrm{~dB}$. The static ISI channel has fixed tap gains and the tap gain powers are the same as those of the Rayleigh fading channel.

The channel estimation length $K^{\dagger}$ is set to the guard interval length $N_{g}$. The actual maximum channel delay spread $K$ for the considered multipath channels is 61 . Since the designed maximum channel delay spread $K^{\prime}$ should be at least equal to or larger than $K$, we set $K^{\prime}=64$. In order to keep the channel impulse response within the channel estimation length $K^{\dagger}$, both $\lambda_{c}+K^{\prime}$ and $\lambda_{f}+K^{\prime}$ should be smaller than $K^{\dagger}$. Hence, we use $\lambda_{c}=30$. Due to the better timing estimation in the fine stage, $\lambda_{f}$ can be set to be smaller than $\lambda_{c}$. The selection of the value of $\lambda_{f}$ is described in our proposed timing performance measure which will be described in Section V-C. In brief, $\lambda_{f}$ can be chosen from the interference-free interval which is shown in our proposed timing performance measure. Based on the above reasons, we set $\lambda_{f}=20$. We estimate that the coarse normalized frequency-offset error would be less than 0.01 for moderate and high SNR values. Hence, we set $F$ to a little larger value 0.05 . Since $2 J+1$ frequency-offset trial points with a resolution of $F / J$ subcarrier spacing are evaluated in the fine stage, a larger value of $J$ can give a slightly better frequency estimation performance, but with a higher complexity. Since we also interpolate as in (35), a frequency resolution of 0.005 is a reasonable value. Hence, we use the corresponding $J$ value of 10 . We evaluated several trial values $0.8,0.4,0.2,0.05,0.01$ for $\eta_{10}$, the channel tap selection threshold at the SNR value of $10 \mathrm{~dB}$. Since $\eta_{10}=0.2$ gives the best fine-timing estimation variance 

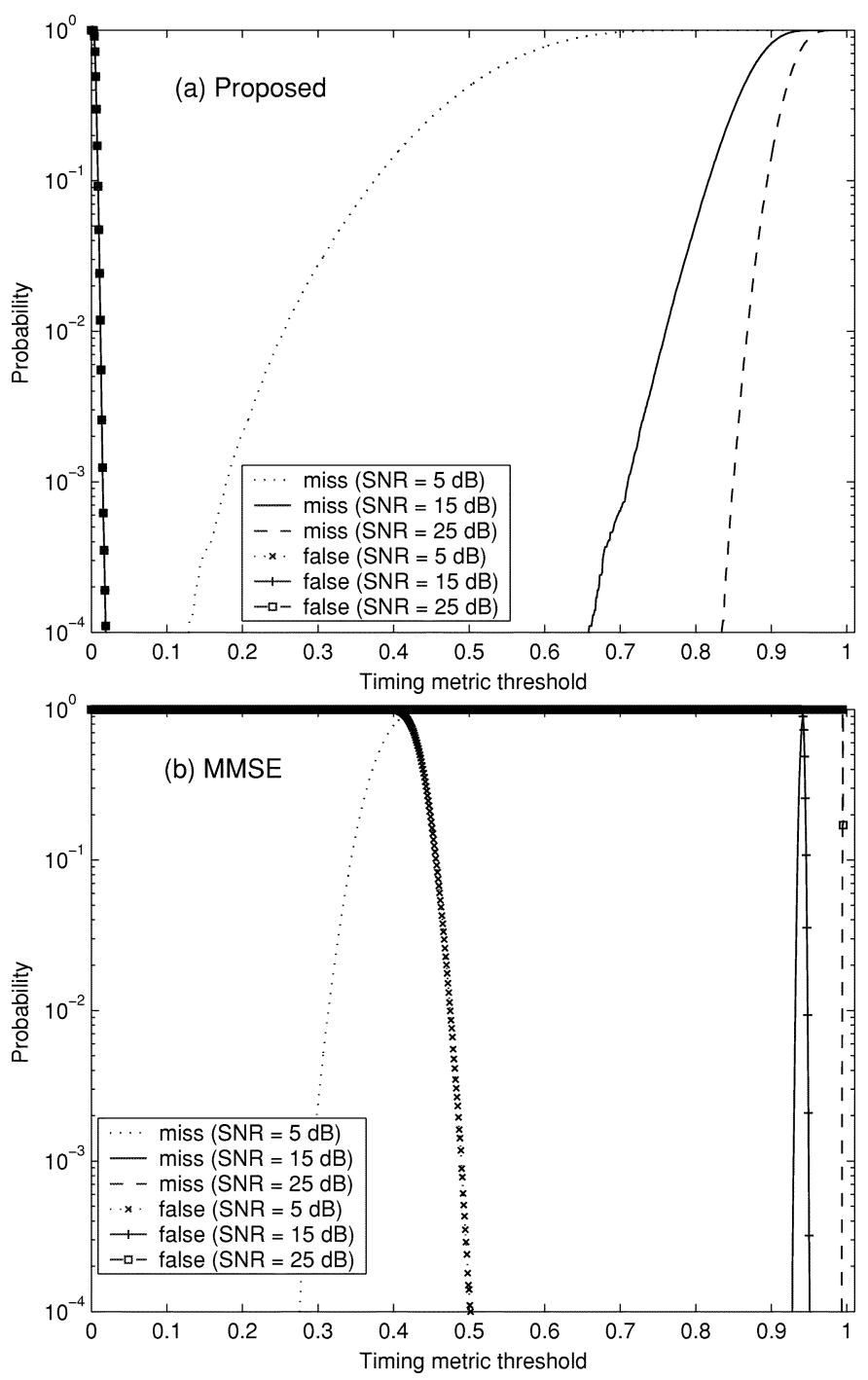

Fig. 5. (a) Sync detection performance of the proposed method. (b) Sync detection performance of the MMSE approach.

in the multipath Rayleigh fading channel, we use $\eta_{10}=0.2$ for the SNR value of $10 \mathrm{~dB}$ throughout our simulations.

\section{B. Missed Detection and False Detection Probabilities}

The missed detection and false detection probabilities of the proposed approach and those associated with the MMSE approach in the 16-tap Rayleigh fading channel described above, obtained from $10^{5}$ simulation runs, are presented in Fig. 5(a) and (b), respectively. Since a burst-mode transmission is considered in the simulations, the false detection probability is evaluated when noise only is present. Hence, the corresponding $1 / \mathrm{SNR}$ values for the false detection probability correspond to the variances of the noise. In evaluating the missed detection probability, the training symbol is preceded by noise samples and followed by an OFDM data symbol. For the MMSE approach, the timing metric of $1-\mathcal{V}(d)$, where $\mathcal{V}(d)$ was given in (9), is used for convenience. The false detection probability curves of the proposed approach are the same for different values of the SNR, as seen in Fig. 5(a), and they are well separated from the missed detection probability curves in the figure. Hence,

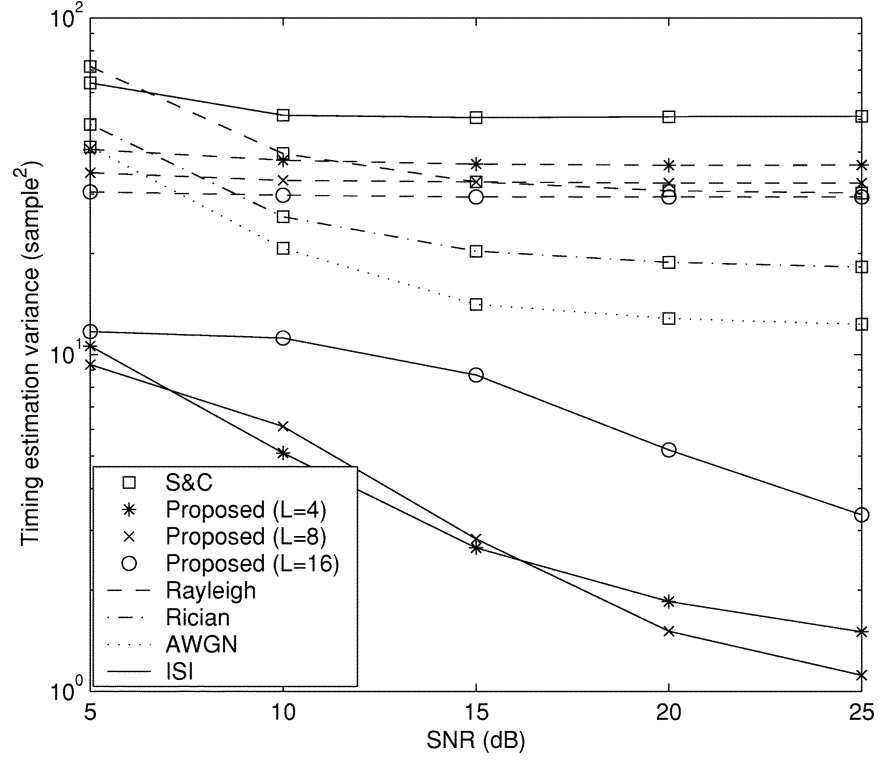

Fig. 6. Timing estimation variance in different channel environments. (No timing offset variations are observed for the proposed method in AWGN and in the multipath Rician fading channels considered, and, hence, the corresponding results are not included in the figure.)

the proposed approach gives a very robust sync detection performance. On the other hand, the false detection probabilities of the MMSE approach depend on the value of the SNR. The higher the SNR, the worse the false detection performance of the MMSE approach. Furthermore, the MMSE approach does not have a timing metric threshold, where both the false detection and the missed detection probabilities are low. Hence, the MMSE approach is associated with a poor sync detection capability. Similarly, the unnormalized timing metrics from [20] are expected to have a poor sync detection capability due to the reason described in Section IV-A.

\section{Timing Synchronization Performance}

Timing estimation performance is often evaluated in terms of the estimator variance. Fig. 6 shows the timing offset variances of S\&C (with $90 \%$ maximum point averaging ${ }^{1}$ ) and the proposed approach (coarse timing only) for $L=4,8$, and 16 . It shows the effect of the proposed training symbol pattern on the timing synchronization performance. For a Rician fading channel and AWGN channel, $d_{\max }$ from (17) of the proposed approach is observed to be always at the correct timing point while there are relatively substantial timing offset variations for S\&C. For the static ISI channel, the proposed approach has some timing offset errors, but they are much smaller than S\&C. From the Rician fading and static ISI channel results, it is noted that if the first channel tap is much larger than the other taps, the proposed approach's $d_{\max }$ can always lock to the first tap correctly. The scheme proposed by $\mathrm{S} \& \mathrm{C}$ also shows a better timing estimation performance in the Rician fading channel than the static ISI channel. For the Rayleigh fading channel, the proposed approach has better timing estimation variance at low

${ }^{1}$ It means that the maximum timing metric point is first found, and then, on each side of the maximum metric point, a timing point having $90 \%$ of the maximum metric is found. The timing estimate is given by the mid point of the two $90 \%$ maximum points. 


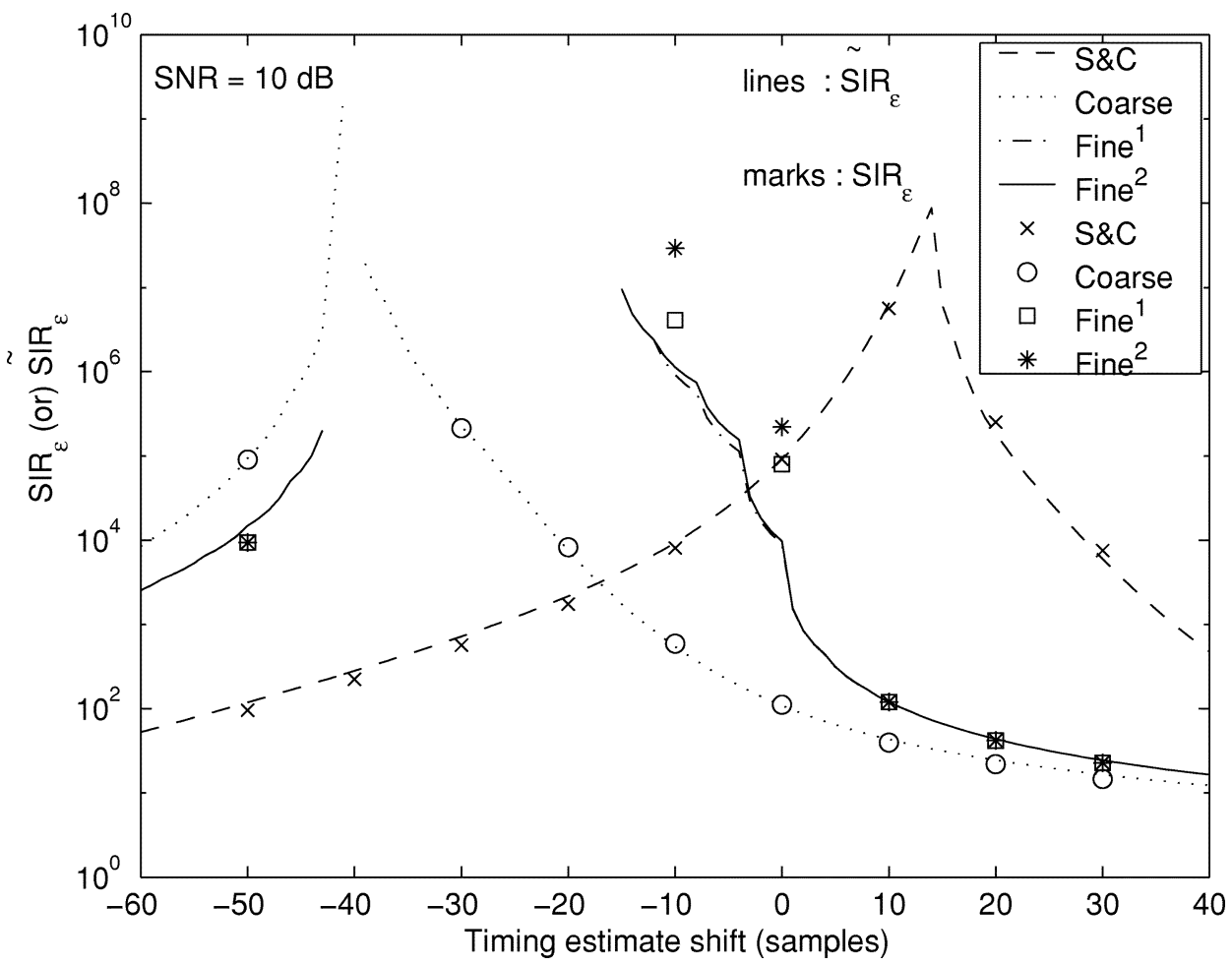

Fig. 7. SIR average interference power ratio and its approximate version versus timing estimate shift in the 16-tap Rayleigh fading channel at an SNR value of $10 \mathrm{~dB}$.

SNR values but comparable variance at high SNR values when compared with S\&C.

For AWGN, static ISI, and Rician channels, the timing estimation variance with reference to the first channel tap has some meaning since the first tap represents the desired timing point. However, for the Rayleigh fading channel, all channel tap gains are time-varying, and missing the first tap with negligible gain may not result in a performance degradation. Hence, the timing estimation variance with reference to the first channel tap may not represent a good performance measure in this case. Moreover, for OFDM systems, as long as the timing estimate is within the ISI-free guard interval, the timing offset, regardless of its value, will not degrade the system performance. Only when the timing estimate falls outside the ISI-free guard interval, interference will be introduced. Hence, a more performance-oriented approach of evaluating the timing synchronization performance for a particular OFDM system would be the measure of interference power caused by the timing offsets. For each timing offset $\varepsilon$, the corresponding normalized interference power $I(\varepsilon)$, normalized by the signal power, can be calculated as

$$
I(\varepsilon)=\frac{\sigma_{\varepsilon}^{2}}{\alpha^{2}(\varepsilon)}
$$

where $\alpha(\varepsilon)$ and $\sigma_{\varepsilon}^{2}$ are given by (5) and (6), respectively.

Let the distribution of the timing offset estimates for the considered system and channel be $P(\varepsilon)$. The average normalized interference power caused by timing offset errors can then be given by

$$
\mathcal{I}_{\varepsilon}=\sum_{\varepsilon} P(\varepsilon) I(\varepsilon)
$$

The calculation of $I(\varepsilon)$ requires an instantaneous channel response (or instantaneous channel power gains) [see (5), (6), and (36)]. Furthermore, the interference power can depend on the timing estimate shift $\lambda$. Note that $\lambda=-\lambda_{c}$ in (17). Hence, it is not so easy to evaluate $\mathcal{I}_{\varepsilon}$ for different parameter settings of the timing estimator such as different timing estimate shifts $(\lambda)$. One way to circumvent this is to approximate $I(\varepsilon)$ calculation by $\tilde{I}(\varepsilon)$ which uses the channel power delay profile instead of the instantaneous channel power gains.

By assuming that the timing estimate is independent of the instantaneous channel response, ${ }^{2}$ an approximation of $\mathcal{I}_{\varepsilon}(\lambda)$ for a timing estimate shift $\lambda$, denoted by $\tilde{\mathcal{I}}_{\varepsilon}(\lambda)$, can be calculated as

$$
\tilde{\mathcal{I}}_{\varepsilon}(\lambda)=\sum_{\varepsilon} P(\varepsilon-\lambda) \cdot \tilde{I}(\varepsilon) .
$$

From the above equation, the optimum timing estimate shift for a considered system and channel can be obtained by

$$
\lambda_{\text {opt }}=\arg \min _{\lambda}\left(\tilde{\mathcal{I}}_{\varepsilon}(\lambda)\right) .
$$

Let us define the average signal to timing-error-induced interference power ratio $\operatorname{SIR}_{\varepsilon}=1 / \mathcal{I}_{\varepsilon}$ and its approximate version $\widetilde{\operatorname{SIR}}_{\varepsilon}=1 / \tilde{\mathcal{I}}_{\varepsilon}$. In Fig. 7 , the $\widetilde{\operatorname{SIR}}_{\varepsilon}$ versus timing estimate shifts are plotted for $\mathrm{S} \& \mathrm{C}$ and the proposed method $(L=4$, FD training) for the Rayleigh fading channel at the SNR value of $10 \mathrm{~dB}$. Also included in the figure are the $\operatorname{SIR}_{\varepsilon}$ values for the timing estimate shifts of $-50,-40, \ldots, 30$ samples. It can be observed that $\operatorname{SIR}_{\varepsilon}$ and $\widetilde{\operatorname{SIR}_{\varepsilon}}$ are almost the same for $\mathrm{S} \& \mathrm{C}$ and the coarse-timing stage of the proposed method, thus indicating

${ }^{2}$ Strictly speaking, this is not true. 
(a) FD

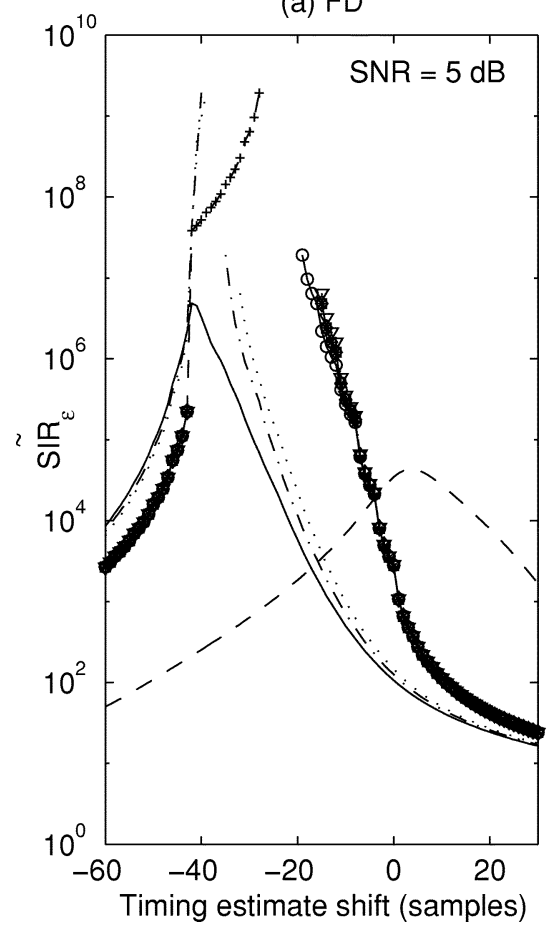

(b) TD

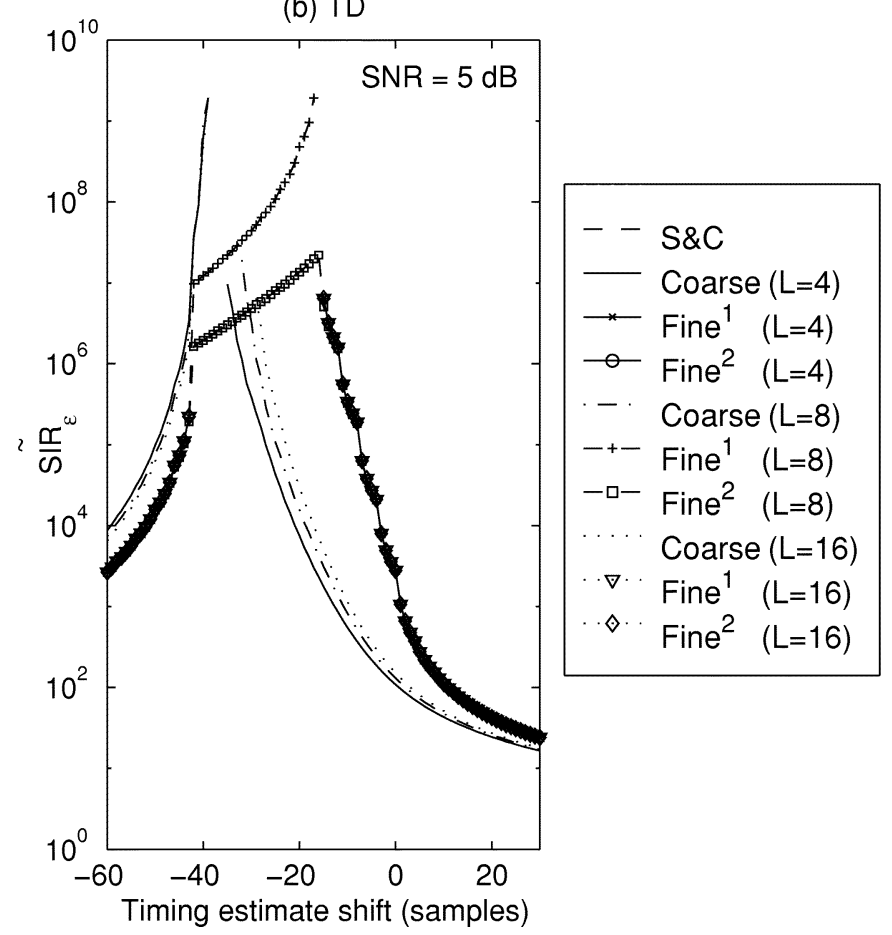

Fig. 8. Timing synchronization performance in terms of $\widetilde{\mathrm{SIR}_{\varepsilon}}$ in the 16-tap Rayleigh fading channel at an SNR value of $5 \mathrm{~dB}$.

that the proposed approximate performance measure is a close approximation.

For the proposed fine-timing stage with one iteration ("Fine ${ }^{1 ")}$ ) and two iterations ("Fine ${ }^{2}$ "), the actual values $\mathrm{SIR}_{\varepsilon}$ at the timing estimate shifts of -10 and 0 samples are greater than the approximate values $\widetilde{\mathrm{SIR}_{\varepsilon}}$ by some amount. This can be explained as follows. The approximate expression $\widetilde{\mathrm{SIR}_{\varepsilon}}$ is under the assumption that the timing estimate is independent of the instantaneous channel response. However, the proposed fine stage utilizes the information from the channel estimation. Hence, the above assumption is not justified. It can be observed that at these timing estimate shifts, the interference is caused by timing offsets greater than zero. For the proposed fine-timing stage, this will most likely happen when the first channel tap gain is quite small. Consequently, the introduced interference which depends on the first channel tap gain would be smaller than the interference used in $\widetilde{\operatorname{SIR}}_{\varepsilon}$ where the power delay profile is used. Despite some differences in values, the plots of $\operatorname{SIR}_{\varepsilon}$ and $\widetilde{\operatorname{SIR}}_{\varepsilon}$ for the fine stage show the same trend. Hence, it still gives useful information on what timing estimate shift would be suitable.

Fig. 7 demonstrates the importance of proper timing shift setting. It can also be observed that comparing two timing estimators, each with a fixed timing estimate shift, will not give the general performance of the timing estimators. Each timing estimator has its own optimum timing estimate shift value(s). The optimum timing estimate shift for $\mathrm{S} \& \mathrm{C}$ in this case is at 14 samples, which still introduces some interference. For the proposed coarse-timing case, the optimum shift is at -40 samples where no interference is introduced. For the fine-timing stage, the in- terference-free timing shift is within the interval from -42 to -16 samples.

It should be mentioned that although the timing estimation variance of $L=16$ (coarse) case for the static ISI channel in Fig. 6 is larger than $L=4$ and 8 cases, evaluating $\widetilde{\operatorname{SIR}}_{\varepsilon}$ versus timing estimate shifts plot at the SNR value of $10 \mathrm{~dB}$ gives an interference-free interval of 35 samples $(-46$ to -12$)$ for both $L=16$ and 8 cases, 28 samples $(-45$ to -18$)$ for $L=4$ case. Due to space limitation, this is not shown. From this fact, it can be stated that the timing estimation variance in OFDM system may not always result in a meaningful performance measure.

In Figs. 8-10, the timing estimation performance in the Rayleigh fading channel in terms of $\widetilde{\mathrm{SIR}_{\varepsilon}}$ are presented for $\mathrm{S} \& \mathrm{C}$ and the proposed method. Both FD training and TD training are evaluated for the proposed method with $L=4$, 8 , and 16 cases. A close observation of these figures indicates that the proposed coarse-timing stage has better performance than S\&C, particularly at low SNR values. At high SNR values, $\mathrm{S} \& \mathrm{C}$ has a comparable performance to the proposed coarse-timing stage. For example, at an SNR value of $25 \mathrm{~dB}$, $\mathrm{S} \& \mathrm{C}$ has an interference-free timing shift interval of four samples (15 to 18 ), and the proposed coarse-timing stage has five samples $(-41$ to -37$)$ for FD and nine samples $(-42$ to -34) for TD. The coarse-timing stage with a larger value of $L$ has a larger interference-free interval. It can be ascribed to the sharper timing metric trajectory for the larger $L$ value. The fine-timing stages ("Fine ${ }^{1 "}$ and "Fine" ${ }^{2}$ ") are observed to give significant improvement over the coarse-timing stage. Although the "Fine" ${ }^{2}$ " case has generally a slight improvement over the "Fine ${ }^{1}$ " case, the performance gain is not significant and may not be justified for the associated complexity cost. 
(a) FD

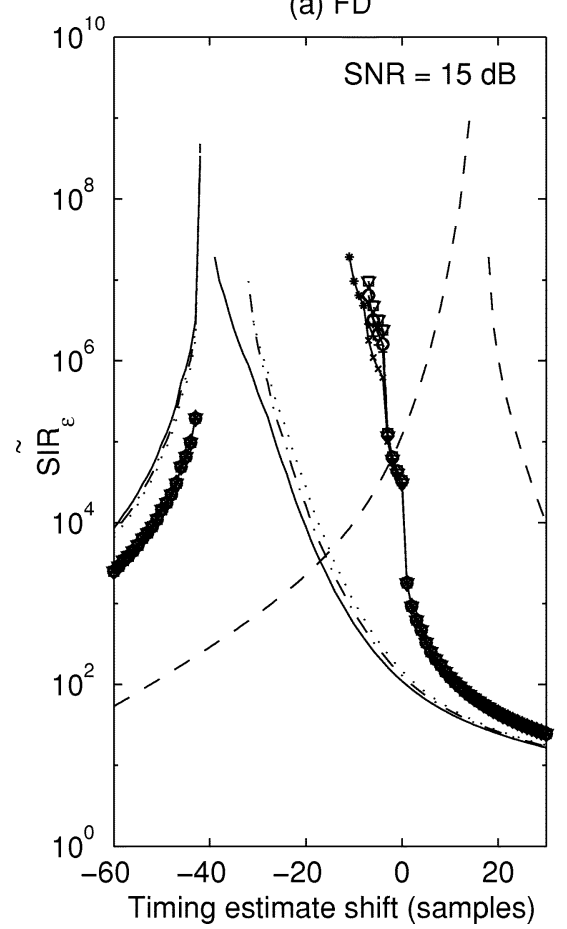

(b) TD

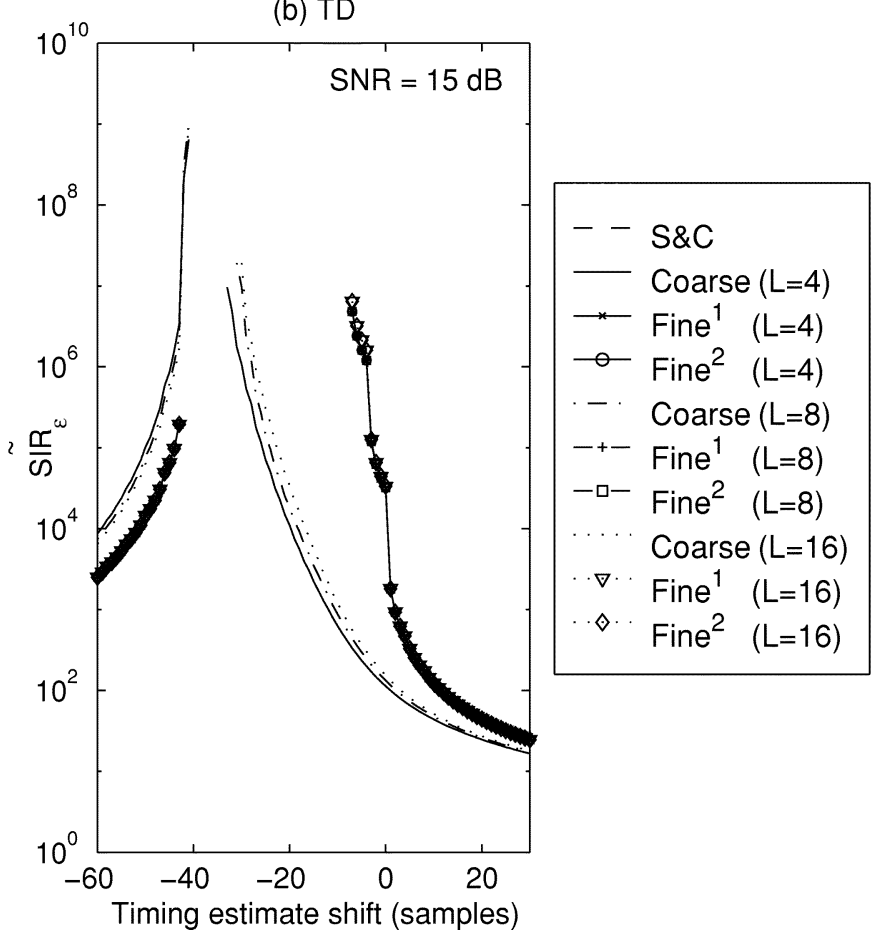

Fig. 9. Timing synchronization performance in terms of $\widetilde{\mathrm{SIR}_{\varepsilon}}$ in the 16-tap Rayleigh fading channel at an SNR value of $15 \mathrm{~dB}$.
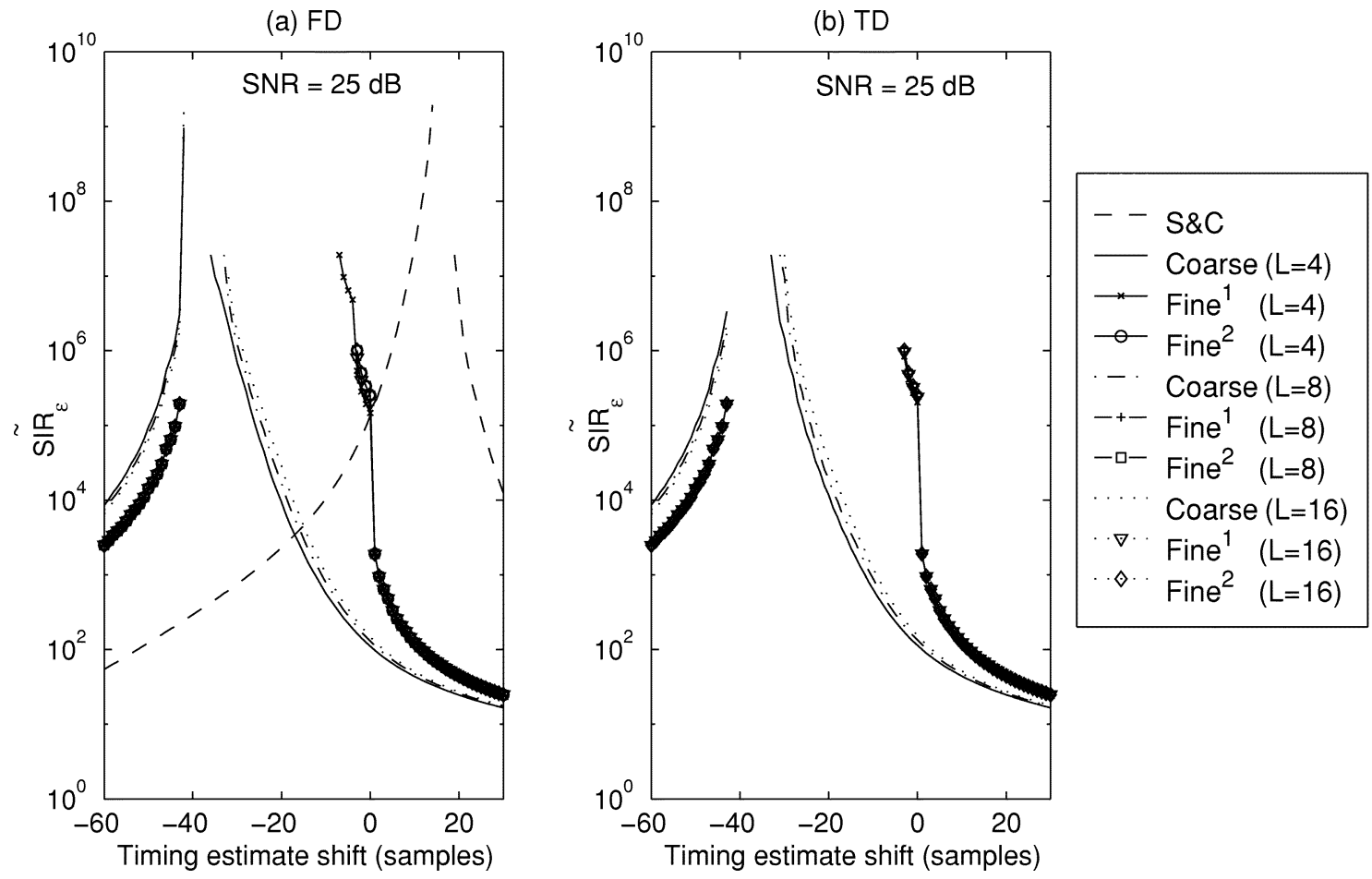

Fig. 10. Timing synchronization performance in terms of $\widetilde{S I R}_{\varepsilon}$ in the 16-tap Rayleigh fading channel at an SNR value of $25 \mathrm{~dB}$.

The fine stages with different $L$ values have almost the same performance, except at an SNR value of $5 \mathrm{~dB}$, where the $L=8$ case has some degradation. This fact indicates that the channel estimation at the fine-timing stage depends on the $L$ value or the training symbol pattern. Since the training symbol is of repetitive structure with some sign conversion, the channel estimate can be a quite different one, rather than a delayed version, in the presence of a large delay timing offset. Hence, it can result in a wrong selection of the first channel tap and introduce some timing error at the fine stage. This occasional occurrence is observed in the simulation of the $L=8$ case at an SNR value of $5 \mathrm{~dB}$. In particular, "Fine" ${ }^{1}$ " stage of $L=8$ case with FD 
(a)

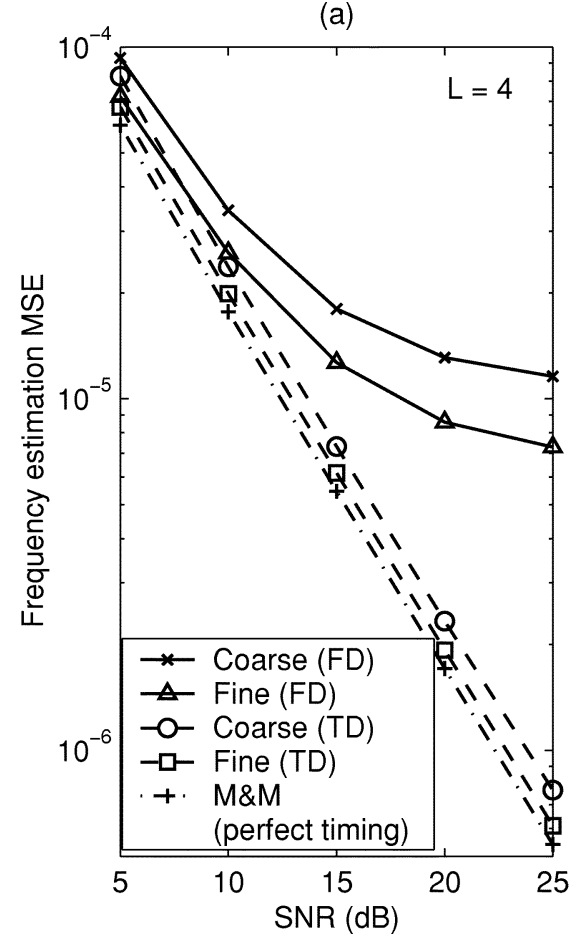

(b)

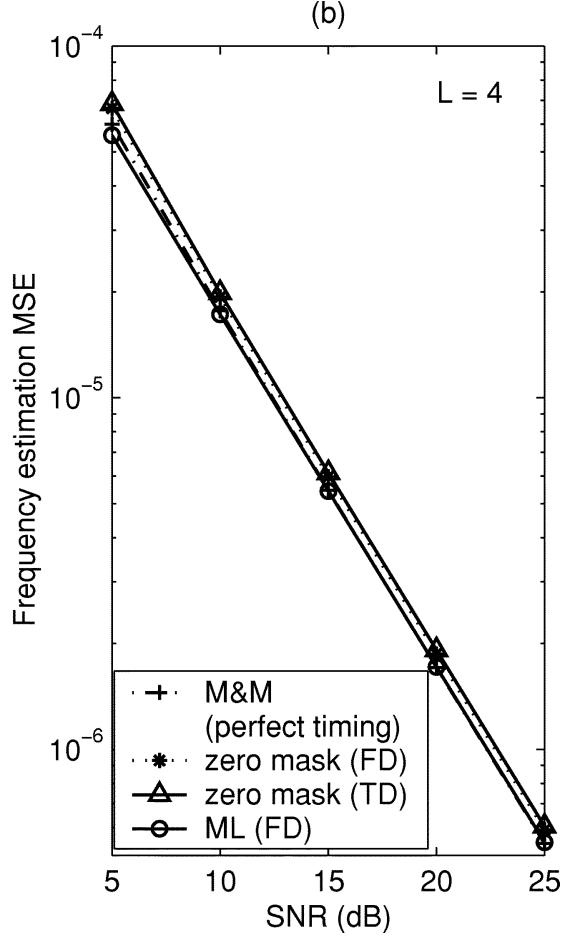

Fig. 11. Frequency estimation MSE performance of the proposed approaches with $L=4$ in the 16-tap Rayleigh fading channel.

training shows such an occurrence but "Fine ${ }^{2}$ " stage is observed to be able to correct the corresponding timing error. However, "Fine ${ }^{2}$ " stage of $L=8$ case with TD training is unable to correct it. Nevertheless, all these cases still have better performance than S\&C. It is also observed from Figs. 8-10 that TD training has a slightly better performance than FD training for all cases except the fine stage of $L=8$ case at an SNR value of $5 \mathrm{~dB}$.

\section{Performance of Frequency Synchronization}

The frequency synchronization performance can be evaluated by the average normalized interference power $\mathcal{I}_{v}$ (normalized by the signal power) caused by frequency estimation errors. The normalized interference power $I_{v}\left(\Delta_{v}\right)$ caused by a normalized (by subcarrier spacing) frequency estimate error $\Delta_{v}$ can be approximated by [21]

$$
I_{v}\left(\Delta_{v}\right) \simeq \frac{\pi^{2}}{3} \cdot \Delta_{v}^{2}
$$

Hence, $\mathcal{I}_{v}$ can be calculated from the mean square error (MSE) of the normalized frequency-offset estimate $\mathrm{mse}_{v}$ as

$$
\mathcal{I}_{v} \simeq \frac{\pi^{2}}{3} \cdot \operatorname{mse}_{v} .
$$

It is noted that the frequency-offset estimation MSE directly translates into the average interference power caused by the frequency estimation errors while the variance of the timing offset estimate does not directly translate into the interference power caused by timing estimation errors. In the following, the frequency estimation MSE will be used as the performance measure for the frequency synchronization.
Figs. 11 and 12 show the frequency estimation performance in the Rayleigh fading channel. The performance of M\&M [12] using a training symbol with 16 identical parts (no sign flipping) under perfect timing synchronization is included as a reference. For the proposed scheme, the results are presented for the cases of coarse frequency estimation ("Coarse"), fine frequency estimation without zero masking ("Fine"), fine frequency estimation with zero masking ("zero mask"), and ML frequency estimation ("ML"). Only the results of the fine stage with one iteration are presented since no noticeable difference is observed between one and two iterations.

All the fine stages have performance improvement over the corresponding coarse stages. However, all fine cases which do not consider the interference effect have some performance degradation if compared with $M \& M$ with perfect timing. An interesting observation from the results of "Fine" case with different $L$ values is that the different training symbol patterns have different impacts on the frequency estimation performance. As previously mentioned, the training symbol pattern can introduce some interference in the frequency estimation in a dispersive channel. This interference seems to be larger for the training symbol pattern of the $L=8$ case, particularly with FD training, as can be observed in Fig. 12(a). Interestingly, TD training is much more robust to this kind of interference than FD training except for the $L=8$ case at an SNR value of $5 \mathrm{~dB}$. An intuitive reason for the better performance of TD training is that the TD training sample energies are distributed evenly, and so do the average noise samples energies. Hence, the energy distribution of TD training is matched to that of the noise, while FD training does not. 
(a)

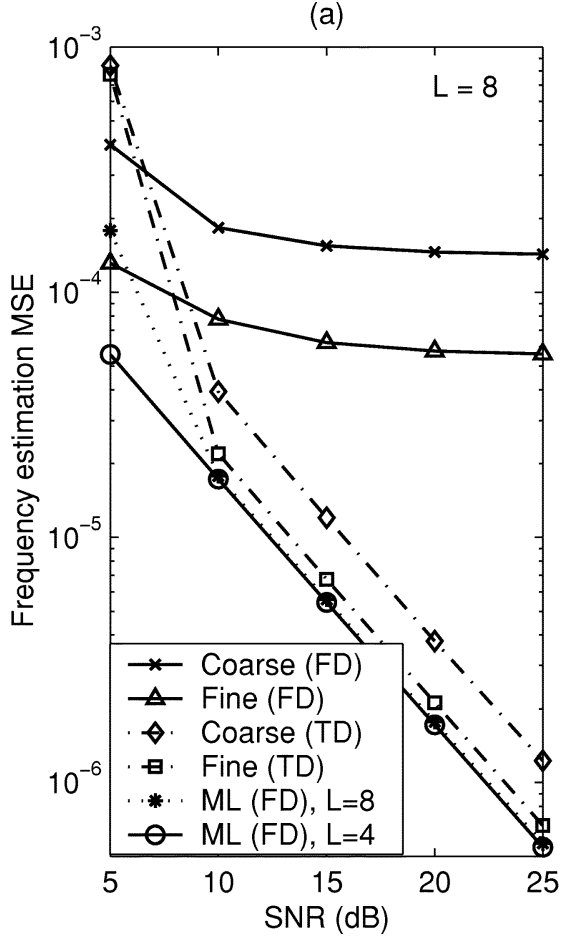

(b)

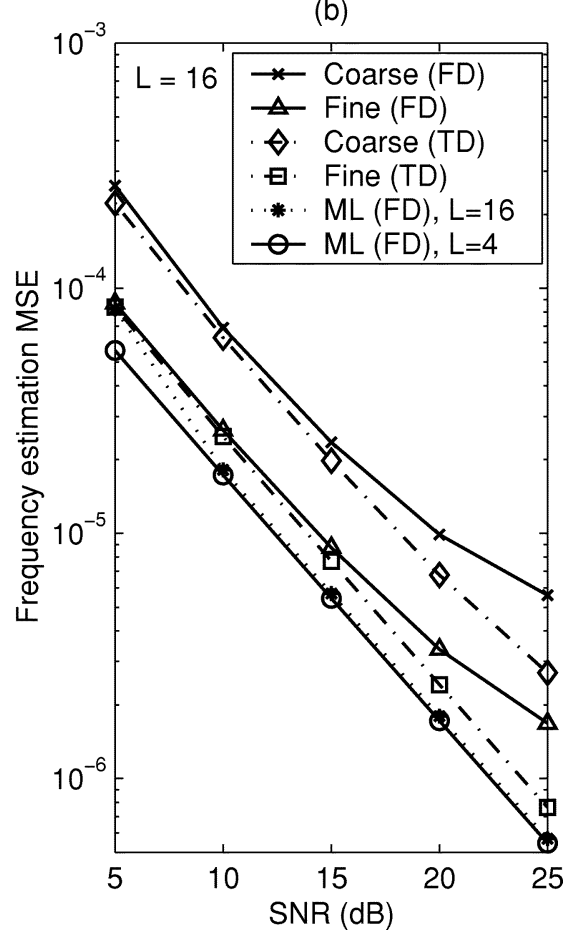

Fig. 12. Frequency estimation MSE performance of the proposed approaches with $L=8$ and 16 in the 16-tap Rayleigh fading channel.

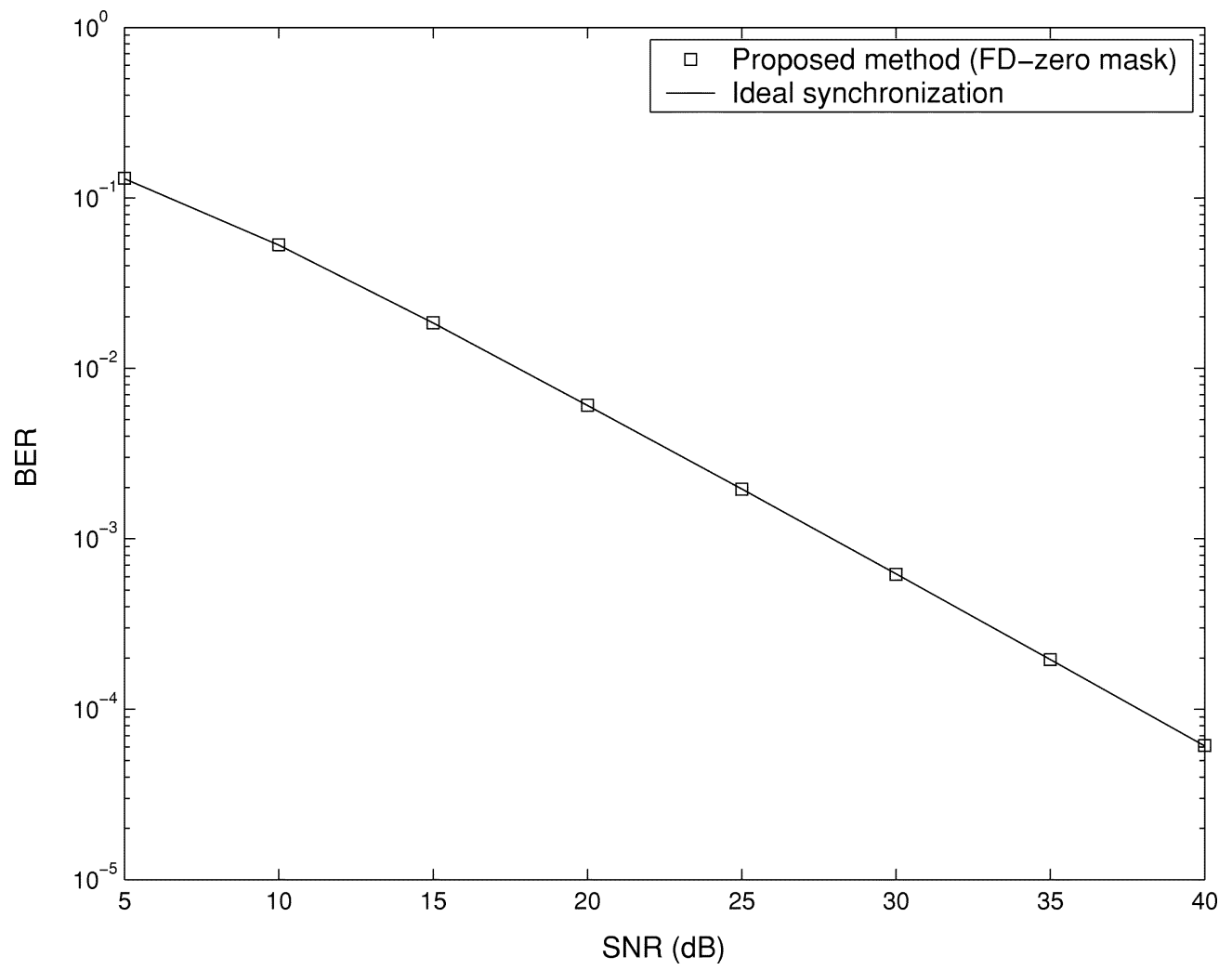

Fig. 13. BER performance comparison between the ideally synchronized system and the system using the proposed method ( $L=4$, FD training, zero masking) in the 16-tap Rayleigh fading channel.

In Fig. 11(b), the performance of the fine frequency estimation approaches, which consider the interference effect, are pre- sented. In fact, the ML approach does not suffer from the interference effect. Both zero-masking approaches with FD and 
TD training have almost the same performance and this performance is very close to the performance of $M \& M$ with perfect timing. Hence, zero masking appears to be an effective approach for the system which uses only one FD training (with possible sign flipping of the repetitive parts) for both timing and frequency synchronization and the length of one repetitive part is quite larger than the maximum channel delay spread. The ML approach has even a better performance than M\&M, particularly at low SNR values. However, in Fig. 12, the ML schemes with $L=8$ and 16 cases show some performance degradation at an SNR value of $5 \mathrm{~dB}$ if compared with $L=4$ case. In fact, this is due to the occasional occurrence of the coarse frequency-offset estimates for which the search space $\hat{v}_{c}-F \leq \tilde{v} \leq \hat{v}_{c}+F$ is not close enough to the actual frequency offset where we note that $F=0.05$ is used in the simulation. To confirm this fact, the ML approach for $L=8$ and 16 cases at an SNR value of $5 \mathrm{~dB}$ are evaluated for $F=1.5$, and $J=10$. The results obtained (not shown in the figure) are almost the same as that of ML with $L=4$.

In general, TD training outperforms FD training. If only one training is used for both timing and frequency synchronization, the impact of the training symbol pattern can affect the frequency estimation performance. In this case, TD training or FD training with the zero-masking approach can be considered. If complexity is affordable, the ML approach can be considered. If two training symbols are applied, one with the proposed training symbol pattern can be considered for timing synchronization using the proposed timing metric, and the other repetitive training without sign flipping can be considered for the frequency estimation using M\&M. Also in this case, TD training is preferable over FD training.

\section{E. BER Performance}

Fig. 13 presents the BER performance comparison between the system using the proposed method and the system having ideal timing and frequency synchronization with the same channel estimation as in the proposed method. The considered system uses FD training followed by an OFDM data symbol and the channel is the multipath Rayleigh fading channel described previously. In the proposed method, the zero-masking approach is used for fine frequency estimation. For SNR values of $25 \mathrm{~dB}$ and above, 100000 simulation runs were used compared with 10000 simulation runs for the other SNR values. It can be seen that for all SNR values, the proposed method has virtually the same BER performance as the perfectly synchronized system.

\section{CONCLUSION}

A robust symbol-timing and carrier-frequency synchronization method for OFDM systems in multipath fading environments has been presented. The proposed method uses one specifically designed training symbol having a steep rolloff timing metric trajectory. This type of training symbol achieves some improvement in timing estimation for time-varying multipath Rayleigh fading channels and much more improvement in AWGN, Rician fading, and static dispersive channels. The channel estimation based on the designed training symbol is also incorporated to give further improvement in timing and frequency synchronization. This combined approach achieves considerable improvement by removing the time-varying multipath effect on timing synchronization. Using the training symbol of identical parts with different signs, however, can introduce interference in the frequency estimation based on that training symbol. An approach of masking the interfered part with zeros is shown to be an effective way for suppressing the introduced interference in the frequency estimation. An ML frequency estimation which does not suffer from this interference is also described. From the simulation, using TD training is observed to be better than FD training.

For frequency estimation, MSE or frequency-error-introduced interference power reflects the frequency synchronization performance. However, for timing estimation, the plot of signal to (timing-error-induced) average interference power ratio $\operatorname{SIR}_{\varepsilon}$ versus timing estimate shift for the considered system and channel appears to be a more revealing performance measure. A close approximation of this $\mathrm{SIR}_{\varepsilon}$ is also proposed using the channel power delay profile. This approximation also yields a simple approach for finding the optimum setting of the timing estimator.

\section{APPENDIX}

This appendix outlines the main steps in obtaining (31). Define two random variables $D_{n}$ and $D_{h}$ as

$$
\begin{aligned}
D_{n} \triangleq \max _{1}\left\{\left[\hat{h}_{i_{1}-m}, \hat{h}_{i_{1}-m+1}, \ldots, \hat{h}_{i_{1}-1}\right]\right\} \\
i_{1} \text { corresponds to the first actual channel tap } \\
m \text { is integer }>0 \\
D_{h} \triangleq \max _{i}\left\{\hat{h}_{i}\right\} .
\end{aligned}
$$

$D_{n}$ and $D_{h}$ can be approximated as complex Gaussian variables with zero means and variances $\Omega_{n}$ and $\Omega_{h}$, where

$$
\begin{aligned}
& \Omega_{n}=L_{1} \frac{m}{N \cdot \mathrm{SNR}} \\
& \Omega_{h}=L_{2} \sum_{l}\left(\sigma_{h_{l}}^{2}+\frac{1}{N \cdot \mathrm{SNR}}\right) \simeq L_{2} \sum_{l} \sigma_{h_{l}}^{2}=L_{2}
\end{aligned}
$$

where $\sigma_{h_{l}}^{2}$ is the variance of the $l$ th channel tap gain, $\sum_{l} \sigma_{h_{l}}^{2}=$ 1 , and $L_{1}$ and $L_{2}$ are some constants $>0$.

Now, our interest is to find $\operatorname{Prob}\left\{\left|D_{n}\right| \leq \eta \cdot\left|D_{h}\right|\right\}, \eta>0$. By defining another random variable $Z \triangleq\left|D_{n}\right| /\left|D_{h}\right|$, we obtain

$$
\operatorname{Prob}\left\{\left|D_{n}\right| \leq \eta \cdot\left|D_{h}\right|\right\}=\operatorname{Prob}\{Z \leq \eta\}=\frac{\eta^{2}}{\eta^{2}+\Omega_{n} / \Omega_{h}}
$$

Using $\Omega_{n} / \Omega_{h} \propto 1 /(N \cdot \mathrm{SNR})$ in the above equation results in (31). 


\section{REFERENCES}

[1] H. Sari, G. Karam, and I. Jeanclaude, "Transmission techniques for digital terrestrial TV broadcasting," IEEE Commun. Mag., vol. 33, pp. 100-109, Feb. 1995.

[2] U. Reimers, "Digital video broadcasting," IEEE Commun. Mag., vol. 36, pp. 104-110, June 1998.

[3] L. J. Cimini, Jr., J. C. Chuang, and N. R. Sollenberger, "Advanced cellular Internet services," IEEE Commun. Mag., vol. 36, pp. 150-159, Oct. 1998.

[4] T. Pollet, M. Van Bladel, and M. Moeneclaey, "BER sensitivity of OFDM systems to carrier frequency offset and Wiener phase noise," IEEE Trans. Commun., vol. 43, pp. 191-193, Feb./Mar./Apr. 1995.

[5] M. Gudmundson and P. O. Anderson, "Adjacent channel interference in an OFDM system," in Proc. Vehicular Technol. Conf., Atlanta, GA, May 1996, pp. 918-922.

[6] J.-J. van de Beek, M. Sandell, and P. O. Börjesson, "ML estimation of time and frequency offset in OFDM systems," IEEE Trans. Signal Processing, vol. 45, pp. 1800-1805, July 1997.

[7] T. M. Schmidl and D. C. Cox, "Robust frequency and timing synchronization for OFDM," IEEE Trans. Commun., vol. 45, pp. 1613-1621, Dec. 1997.

[8] M. Speth, D. Daecke, and H. Meyr, "Minimum overhead burst synchronization for OFDM based broadband transmission," in Proc. Global Telecommun. Conf., Sydney, Australia, Nov. 1998, pp. 2777-2782.

[9] P. H. Moose, "A technique for orthogonal frequency division multiplexing frequency offset correction," IEEE Trans. Commun., vol. 42, pp. 2908-2914, Oct. 1994.

[10] F. Daffara and O. Adami, "A new frequency detector for orthogonal multicarrier transmission techniques," in Proc. Vehicular Technol. Conf., Chicago, IL, July 1995, pp. 804-809.

[11] H. Nogami and T. Nagashima, "A frequency and timing period acquisition technique for OFDM systems," IEICE Trans. Commun., vol. E79-B, no. 8, pp. 1135-1146, 1996.

[12] M. Morelli and U. Mengali, "An improved frequency offset estimator for OFDM applications," IEEE Commun. Lett., vol. 3, pp. 75-77, Mar. 1999.

[13] M. Speth, F. Classen, and H. Meyr, "Frame synchronization of OFDM systems in frequency selective fading channels," in Proc. Vehicular Technol. Conf., Phoenix, AZ, May 1997, pp. 1807-1811.

[14] L. Hazy and M. El-Tanany, "Synchronization of OFDM systems over frequency selective fading channels," in Proc. Vehicular Technol. Conf., Phoenix, AZ, May 1997, pp. 2094-2098

[15] H. Minn, M. Zeng, and V. K. Bhargava, "On timing offset estimation for OFDM systems," IEEE Commun. Lett., vol. 4, pp. 242-244, July 2000.

[16] B. Yang, K. B. Letaief, R. S. Cheng, and Z. Cao, "Timing recovery for OFDM transmission," IEEE J. Select. Areas Commun., vol. 18, pp. 2278-2290, Nov. 2000.

[17] S. A. Fechtel and H. Meyr, "Improved frame synchronization for spontaneous packet transmission over frequency-selective radio channels," in Proc. PIMRC, The Hague, The Netherlands, 1994, pp. 353-357.

[18] A. Czylwik, "Synchronization for single carrier modulation with frequency domain equalization," in Proc. VTC, Ottawa, ON, Canada, 1998, pp. 2277-2281.

[19] P. R. Chevillat, D. Maiwald, and G. Ungerboeck, "Rapid training of a voiceband data-modem receiver employing an equalizer with fractional-T spaced coefficients," IEEE Trans. Commun., vol. COM-35, pp. 869-876, Sept. 1987

[20] S. H. Müller-Weinfurtner, "On the optimality of metrics for coarse frame synchronization in OFDM: A comparison," in Proc. PIMRC, Boston, MA, 1998, pp. 533-537.

[21] M. Speth, S. A. Fechtel, G. Fock, and H. Meyr, "Optimum receiver design for wireless broad-band systems using OFDM-Part I," IEEE Trans. Commun., vol. 47, pp. 1668-1677, Nov. 1999.

[22] "Broadband Radio Access Networks (BRAN); HIPERLAN Type 2; Physical (PHY) layer technical specification," ETSI, ETSI TS 101475 v1.1.1 (2000-04)

[23] M. J. E. Golay, "Complementary series," IRE Trans. Inform. Theory, vol. IT-7, pp. 82-87, Apr. 1961.

[24] R. D. J. van Nee, "OFDM codes for peak-to-average power reduction and error correction," in Proc. Global Telecommun. Conf., London, U.K., Nov. 1996, pp. 740-744.
[25] S. M. Kay, Fundamentals of Statistical Signal Processing: Estimation Theory. Englewood Cliffs, NJ: Prentice-Hall, 1993.

[26] M. Morelli and U. Mengalli, "Carrier-frequency estimation for transmissions over selective channels," IEEE Trans. Commun., vol. 48, pp. 1580-1589, Sept. 2000.

[27] U. Lambrette, M. Speth, and H. Meyr, "OFDM burst frequency synchronization by single carrier training data," IEEE Commun. Lett., vol. 1, pp. 46-48, Mar. 1997.

[28] T. Keller, L. Piazzo, P. Mandarini, and L. Hanzo, "Orthogonal frequency division multiplex synchronization techniques for frequency-selective fading channels," IEEE J. Select. Areas Commun., vol. 19, pp. 999-1008, June 2001.

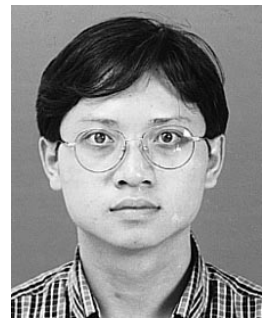

Hlaing Minn (S'99-M'01) received the B.E. degree in electronics from the Yangon Institute of Technology, Yangon, Myanmar, in 1995, the M.Eng. degree in telecommunications from the Asian Institute of Technology (AIT), Bangkok, Thailand, in 1997, and the Ph.D. degree in electrical engineering from the University of Victoria, Victoria, BC, Canada, in 2001.

He was with the Telecommunications Program at the Asian Institute of Technology, as a Laboratory Supervisor during 1998. He was a Research Assistant from 1999 to 2001 and a Research Fellow during 2002 in the Department of Electrical and Computer Engineering at the University of Victoria. Since September 2002, he has been an Assistant Professor with the Erik Jonsson School of Engineering and Computer Science, the University of Texas at Dallas, Richardson, TX. His research interests include wireless communications, signal processing, and error-control coding, detection, and estimation.

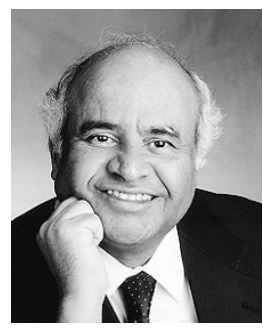

Vijay K. Bhargava (S'70-M'74-SM'82-F'92) received the B.Sc., M.Sc., and Ph.D. degrees from Queen's University, Kingston, ON, Canada in 1970, 1972, and 1974, respectively.

Currently, he is a Professor and Head of the Department of Electrical and Computer Engineering at the University of British Columbia, Vancouver, BC, Canada. Previously, he was with the University of Victoria (1984-2003) and with Concordia University in Montréal (1976-1984). He is a coauthor of the book Digital Communications by Satellite (New York: Wiley, 1981), coeditor of Reed-Solomon Codes and Their Applications (New York: IEEE, 1994), and coeditor of Communications, Information and Network Security (Boston, MA: Kluwer, 2002). His research interests are in multimedia wireless communications.

Dr. Bhargava is very active in the IEEE and was nominated by the IEEE Board of Directors for the Office of IEEE President-Elect. Currently, he serves on the Board of the IEEE Communications Society. He is an Editor for the IEEE TRANSACTIONS ON COMMUNICATIONS and the IEEE TRANSACTIONS ON WiRELESS COMMUNICATIONS. He is a Past President of the IEEE Information Theory Society. He is a Fellow of the B.C. Advanced Systems Institute, Engineering Institute of Canada (EIC), and the Royal Society of Canada. He is a recipient of the IEEE Centennial Medal (1984), IEEE Canada's McNaughton Gold Medal (1995), the IEEE Haraden Pratt Award (1999), the IEEE Third Millennium Medal (2000), and the IEEE Graduate Teaching Award (2002). 


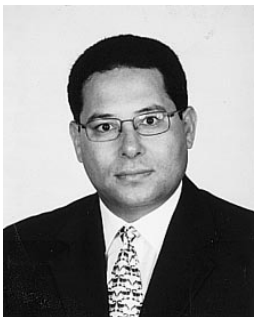

Khaled Ben Letaief (S'85-M'86-SM'97-F'03) received the B.S. degree (with distinction) and the M.S. and Ph.D. degrees from Purdue University, West Lafayette, IN, in 1984, 1986, and 1990, respectively, all in electrical engineering.

From January 1985 and as a Graduate Instructor in the School of Electrical Engineering at Purdue University, he has taught courses in communications and electronics. From 1990 to September 1993, he was a faculty member in the Department of Electrical and Electronic Engineering at the University of Melbourne, Australia, where he was also a Member of the Center for Sensor Signal and Information Systems. Since September 1993, he has been with the Department of Electrical and Electronic Engineering at The Hong Kong University of Science and Technology (HKUST), Hong Kong, where he is now a Professor and Head. His current research interests include wireless and mobile communications, OFDM, space-time processing for wireless systems, multiuser detection, wireless multimedia communications, and CDMA systems.

Dr. Letaief was appointed the founding Editor-in-Chief of the IEEE TRANSACTIONS ON WIRELESS COMMUNICATIONS, in January 2002. He has served on the Editorial Boards of other journals including the IEEE TransaCtions on COMmunications, IEEE Communications Magazine, Wireless Personal Communications, and IEEE JOURNAL ON SELECTED AREAS IN COMMUNICATIONS-WiRELESS SERIES (as Editor-in-Chief). He served as the Technical Program Chair of the 1998 IEEE Globecom Mini-Conference on Communications Theory, held in Sydney, Australia. He is also the Co-Chair of the 2001 IEEE ICC Communications Theory Symposium, held in Helsinki, Finland. He is currently serving as Vice-Chair of the IEEE Communications Society Technical Committee on Personal Communications. He is also currently the Vice chair of Meeting and Conference Committee of the IEEE COMSOC Asia Pacific Board. In addition to his active research activities, he has also been a dedicated teacher committed to excellence in teaching and scholarship. He received the Mangoon Teaching Award from Purdue University in 1990, the Teaching Excellence Appreciation Award by the School of Engineering at HKUST in Spring 1995, Fall 1996, Fall 1997, and Spring 1999, and the Michael G. Gale Medal for Distinguished Teaching (highest university-wide teaching award and only one recipient/year is honored for his/her contributions) 